\title{
Optimal Energy-efficient Source and Relay Precoder Design for Cooperative MIMO-AF Systems
}

\author{
Fabien Héliot, Member, IEEE and Rahim Tafazolli, Senior Member, IEEE
}

\begin{abstract}
Energy efficiency (EE) is a key design criterion for the next generation of communication systems. Equally, cooperative communication is known to be very effective for enhancing the performance of such systems. This paper proposes a breakthrough approach for maximizing the EE of multiple-inputmultiple-output (MIMO) relay-based nonregenerative cooperative communication systems by optimizing both the source and relay precoders when both relay and direct links are considered. We prove that the corresponding optimization problem is at least strictly pseudo-convex, i.e. having a unique solution, when the relay precoding matrix is known, and that its Lagrangian can be lower and upper bounded by strictly pseudo-convex functions when the source precoding matrix is known. Accordingly, we then derive EE-optimal source and relay precoding matrices that are jointly optimize through alternating optimization. We also provide a low-complexity alternative to the EE-optimal relay precoding matrix that exhibits close to optimal performance, but with a significantly reduced complexity. Simulations results show that our joint source and relay precoding optimization can improve the EE of MIMO-AF systems by up to $50 \%$ when compared to direct/relay link only precoding optimization.
\end{abstract}

Index Terms-Energy efficiency, precoding/beamforming, cooperative communication, MIMO, amplify-and-forward.

\section{INTRODUCTION}

Energy efficiency (EE) is one of the eight key figures of merit identified by the international telecommunication union (ITU) for shaping the next generation of communication systems [1]; as such, EE has recently received a surge of interests from the research community [2]-[4], as well as network vendors and operators who perceive EE as an enabler for sustainable communication (both from an economical and environmental perspectives). Equally, relay-based cooperative communication, which is also well-documented [5]-[7], has proved to be very effective for improving the spectral efficiency (SE) or/and the coverage of cellular networks [7], as well as reducing the cost of network deployment [8]. More recently, relays have also been used for improving the EE [9][12]. As a result, relay-based cooperative communication is an integral part of the existing wireless communication standards [13]; it is also foreseen to play a major role for enabling device-to-device communication in the near future [1].

Amongst the various existing relay-based communication strategies (e.g. amplify-and-forward (AF), decode-andforward, compress-and-forward), AF remains one of the most popular strategy given its simplicity and practicality for enabling multi-input multi-output (MIMO) cooperative com-

The authors are with the Institute for Communication Systems, Faculty of Electronics \& Physical Sciences, University of Surrey, Guildford GU2 7XH, UK (e-mail: F.Heliot@Surrey.ac.uk).

We would like to acknowledge the support of the University of Surrey 5GIC (http://www.surrey.ac.uk/5gic) members for this work. munication. As such, MIMO-AF communication has been thoroughly investigated over the last decade, where numerous works have focused on designing precoding/resource allocation techniques for improving the SE, reducing the detection error rate, or reducing the power consumption of MIMO-AF [14]-[22]. With EE becoming one of the key design parameters in wireless communication, more recent works on MIMOAF communication have started to develop precoding/resource allocation techniques for improving the EE of MIMO-AF [10]-[12]. For instance, EE-optimal precoding/resource allocation schemes have been designed in [10], [11] and [12] for the MIMO-AF two-hop and multi-hop scenarios, respectively, where a source node (SN) transmits data to a destination node (DN) via one or more relay nodes (RNs). However, these works do not take into account the direct link (DL) transmission (i.e. SN to DN transmission is neglected) in their precoding design. As far as SE/detection error-based MIMOAF precoding/resource allocation schemes are concerned, the works in [16]-[20] have focused on the same scenario as in this paper, i.e. the cooperative MIMO-AF scenario, where both the relay link (RL) (i.e. $\mathrm{SN}$ to $\mathrm{RN}$ to $\mathrm{DN}$ transmission) and DL transmissions are fully considered. For instance in [16]-[19], which all focus on SE improvement, heuristic precoding/resource allocation methods have been proposed; however, none of these works have provided any closed-form of the optimal precoding matrix at the $\mathrm{SN}$ or $\mathrm{RN}$. Whereas in [20], optimal $\mathrm{SN}$ and $\mathrm{RN}$ precoding matrices have been obtained (in closed form) for minimizing the mean square error (MSE) (i.e. detection error).

In this paper, we go beyond the works in [10], [11] and propose a breakthrough approach for maximizing the EE of cooperative MIMO-AF systems, where we derive both EEoptimal SN and RN precoding matrices. Deriving $\mathrm{SN}$ and RN precoding matrices was also the aim of [16]-[20], but the precoding matrices of [16]-[20] are designed to either maximize the SE or minimize the MSE instead of maximizing the $\mathrm{EE}$, which is an entirely new proposition given the significant difference in optimization problem formulation between our work and these works. In addition, contrary to [16]-[19], we formally prove the optimality of our SN and RN precoders by relying on pseudo-convexity arguments and provide a closedform expression for the EE-optimal $\mathrm{SN}$ precoding matrix. We assume, as in most existing works on MIMO-AF precoding [16]-[20], [22] that full channel state information (CSI), i.e. transmit and receive CSI, is available at both the SN and RN; further practical detailed about CSI acquisition can be found in [21], [22]. Note that a preliminary version of this work is available in [23]; contrary to [23], we prove here the pseudoconvexity/convexity of the main optimization problem, provide 
an EE-optimal relay precoding matrix (instead of suboptimal), and consider power constraints for designing the source and relay precoders.

The rest of the paper is organized as follows. Section II-A first recalls the layout and then defines the achievable sum-rate, power consumption, as well as energy-per-bit consumption of the cooperative MIMO-AF system. Then, Section III introduces the optimization problem that is solved in this paper, i.e. EE-based iterative joint optimization of the $\mathrm{SN}$ and $\mathrm{RN}$ precoding matrices. We first prove that the Lagrangian function associated to this problem is strictly pseudo-convex or convex for a known RN precoding matrix and obtain the EE-optimal SN precoding matrix in closed-form. We then derive lower and upper bounds of the Lagrangian function for a known SN precoding matrix (which are proved to be strictly pseudoconvex or convex functions) and proposed a novel method for obtaining the EE-optimal RN precoding matrix. We also provide a sub-optimal $\mathrm{RN}$ precoding matrix in closed-form. An iterative process based on alternating optimization [24] is finally utilized, as for instance in [10], [16], [20], for jointly optimizing the $\mathrm{SN}$ and $\mathrm{RN}$ precoding matrices; a process that is proved to converge towards a local optimum at each iteration due to the strictly pseudo-convexity/convexity of the SN/RN precoding optimization problem. Next, Section IV provides a performance as well as complexity analysis of our scheme. Simulation results confirm that joint $\mathrm{SN}$ and RN EE-based precoding optimization can improve the EE performance of MIMO-AF systems by up to $50 \%$ when compared to existing EE-optimal precoding schemes such as in [10], [11]. They also indicate that the classic relay precoding structure of [14], which is known to be optimal in various MIMO-AF optimization cases [10], [11], [14], [25], is not optimal here. Conclusions are finally drawn in Section V.

Notation: The following notation is considered throughout the paper. Boldface lowercase letters (e.g. a) denote vectors, boldface uppercase letters (e.g. A) denote matrices, boldface uppercase letters with a hat on top (e.g. $\widehat{\mathbf{A}}$ ) denote diagonal matrices, and $\mathbf{I}_{x}$ denotes a $x \times x$ identity matrix. Whereas the operator $\operatorname{diag}($.$) transforms a vector into a diag-$ onal matrix (e.g. $\operatorname{diag}(\mathbf{a})=\widehat{\mathbf{A}})$. Moreover, $\mathbf{A} \succ \mathbf{0}$ or $\mathbf{A} \succeq \mathbf{0}$ indicates that $\mathbf{A}$ is a positive definite or semi-definite matrix, respectively, and $\mathbf{A}^{\frac{1}{2}}$ represents the Hermitian square root of $\mathbf{A} \succeq \mathbf{0}$. Furthermore, $||,. \operatorname{tr}\{\},. .^{\dagger}$, and.$^{-1}$ are the determinant, trace, conjugate transpose, and generalized inverse (both inverse and pseudo-inverse) matrix operators, respectively. In addition, $\langle.,\rangle_{\mathrm{F}}$ denotes the Frobenius inner product between two matrices, such that $\langle\mathbf{A}, \mathbf{B}\rangle_{\mathrm{F}}=\operatorname{tr}\left\{\mathbf{A}^{\dagger} \mathbf{B}\right\}$. Finally, the notation $[.]_{+}$refers to $\max \{., 0\}$.

\section{CoOperative MIMO-AF EE Framework}

\section{A. System Model}

This paper focuses on the EE/energy consumption of a classic nonregenerative cooperative MIMO system that is composed of three nodes, i.e. a $\mathrm{SN}$, a $\mathrm{RN}$ and a $\mathrm{DN}$, as it is illustrated in Fig. 1. The $\mathrm{SN}, \mathrm{RN}$ and $\mathrm{DN}$ are equipped with $n_{\mathrm{SN}}, n_{\mathrm{RN}}$ and $n_{\mathrm{DN}}$ antennas, respectively.

As in [14], we assume here that the data transmission is performed over two phases of equal duration, such that the

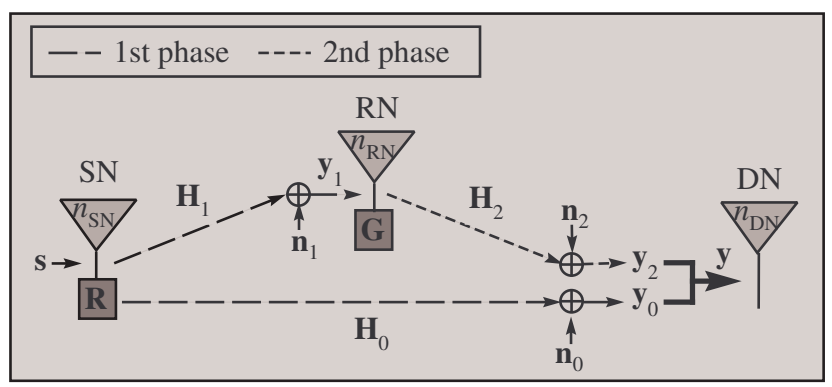

Fig. 1: Nonregenerative cooperative MIMO communication system model.

aggregate mutual information/achievable rate (over two time slots) of the cooperative MIMO-AF system depicted in Fig. 1 can be expressed, in two distinct manners (see equation (6) of $[17])$, as $I(\mathbf{y} ; \mathbf{s})=$

$$
\begin{aligned}
R_{\Sigma}(\mathbf{R}, \mathbf{G}) & =R_{0}(\mathbf{R})+W \log _{2} \mid \mathbf{I}_{n_{\mathrm{DN}}}+\frac{\sigma_{1}^{2}}{\sigma_{2}^{2}} \mathbf{H}_{2} \mathbf{G} \dot{\Upsilon}(\mathbf{R}) \mathbf{G}^{\dagger} \mathbf{H}_{2}^{\dagger} \\
& \times \boldsymbol{\Omega}(\mathbf{G})^{-1} \mid \text { or } \\
R_{\Sigma}(\mathbf{R}, \mathbf{G}) & =W \log _{2}\left|\mathbf{I}_{n_{\mathrm{SN}}}+\mathbf{R}^{\dagger} \dot{\boldsymbol{\Psi}}(\mathbf{G}) \mathbf{R}\right|
\end{aligned}
$$

where

$$
\begin{aligned}
& R_{0}(\mathbf{R})=I\left(\mathbf{y}_{0} ; \mathbf{s}\right)=W \log _{2}\left|\mathbf{I}_{n_{\mathrm{DN}}}+\sigma_{0}^{-2} \mathbf{H}_{0} \mathbf{R} \mathbf{R}^{\dagger} \mathbf{H}_{0}^{\dagger}\right| \\
& \dot{\boldsymbol{\Upsilon}}(\mathbf{R})=\sigma_{1}^{-2} \mathbf{H}_{1} \mathbf{R}\left(\mathbf{I}_{n_{\mathrm{SN}}}+\sigma_{0}^{-2} \mathbf{R}^{\dagger} \mathbf{H}_{0}^{\dagger} \mathbf{H}_{0} \mathbf{R}\right)^{-1} \mathbf{R}^{\dagger} \mathbf{H}_{1}^{\dagger}, \\
& \mathbf{\Omega}(\mathbf{G})=\mathbf{I}_{n_{\mathrm{DN}}}+\frac{\sigma_{1}^{2}}{\sigma_{2}^{2}} \mathbf{H}_{2} \mathbf{G} \mathbf{G}^{\dagger} \mathbf{H}_{2}^{\dagger}, \text { and } \\
& \dot{\boldsymbol{\Psi}}(\mathbf{G})=\sigma_{0}^{-2} \mathbf{H}_{0}^{\dagger} \mathbf{H}_{0}+\sigma_{2}^{-2} \mathbf{H}_{1}^{\dagger} \mathbf{G}^{\dagger} \mathbf{H}_{2}^{\dagger} \mathbf{\Omega}(\mathbf{G})^{-1} \mathbf{H}_{2} \mathbf{G H}_{1} .
\end{aligned}
$$

In addition $W$ is the channel bandwidth, $\mathbf{R} \in \mathbb{C}^{n_{\mathrm{SN}} \times n_{\mathrm{SN}}}$ and $\mathbf{G} \in \mathbb{C}^{n_{\mathrm{RN}} \times n_{\mathrm{RN}}}$ are the $\mathrm{SN}$ and $\mathrm{RN}$ precoding matrices, respectively. Moreover, $\mathbf{H}_{0} \in \mathbb{C}^{n_{\mathrm{DN}} \times n_{\mathrm{SN}}}, \mathbf{H}_{1} \in \mathbb{C}^{n_{\mathrm{RN}} \times n_{\mathrm{SN}}}$, and $\mathbf{H}_{2} \in \mathbb{C}^{n_{\mathrm{DN}} \times n_{\mathrm{RN}}}$ model the $\mathrm{SN}$ to $\mathrm{DN}, \mathrm{SN}$ to $\mathrm{RN}$, and $\mathrm{SN}$ to RN MIMO channels, correspondingly. Furthermore, $\sigma_{0}^{2}, \sigma_{1}^{2}$ and $\sigma_{2}^{2}$ are the variances of the Gaussian noise at the DN (SN-DN link), RN and DN (SN-RN-DN link), respectively.

\section{B. Power Consumption Model and EE-SE Trade-off}

Given that the transmission between the SN and DN occurs in two phases, the way in which power is consumed by each node in each phase can be different. For instance, in the first phase, the SN transmits data to both the RN and DN, which receive it; whereas in the second phase, only the $\mathrm{RN}$ transmits to the DN, such that the $\mathrm{SN}$ is idle (sleep mode). By assuming that three power consumption modes are available for each node, i.e. transmission, $P^{\mathrm{Tx}}$, reception, $P^{\mathrm{Rx}}$, and sleep, $P^{\mathrm{Sl}}$, the total consumed power (over two phases) of the cooperative MIMO-AF system in Fig. 1 can hence be modeled as

$$
P_{\Sigma}=\left(P_{\mathrm{SN}}^{\mathrm{Tx}}+P_{\mathrm{RN}}^{\mathrm{Rx}}+P_{\mathrm{DN}}^{\mathrm{Rx}}\right)+\left(P_{\mathrm{SN}}^{\mathrm{Sl}}+P_{\mathrm{RN}}^{\mathrm{Tx}}+P_{\mathrm{DN}}^{\mathrm{Rx}}\right) .
$$

In transmission mode, a node consumes power for preparing (e.g. baseband processing, RF transceiver chain) and sending the information (power amplifier). According to [4], [26], [27], 
the power consumption of common communication equipments/devices in a cooperative MIMO-AF system, e.g. BS, $\mathrm{RN}$ or user equipment (UE), is linearly dependent with their transmit power when transmitting. Hence, it can be expressed via a generic linear MIMO power model [28]

$$
P^{\mathrm{Tx}}=\Delta . P .+n .^{\mathrm{CipA}}+P^{\mathrm{Ci}},
$$

where $P$. represents the transmit power, $\Delta$. accounts for the inefficiency of the transmitting node power amplifier, $n$. is the number of transmit antennas, $P^{\text {CipA }}$ models the circuit power consumption scaling with $n$. (e.g. RF transceiver chain power consumption), and $P^{\mathrm{Ci}}$ models the other types of circuit power consumption (e.g. DC-DC conversion, baseband processing). In reception mode, the receiving node consumes power for receiving (e.g. RF transceiver chain) and processing the information (e.g. baseband processing), such that

$$
P^{\mathrm{Rx}}=\varsigma\left[n .^{\mathrm{CipA}}+P^{\mathrm{Ci}}\right],
$$

where $0 \leq \varsigma \leq 1$ given that reception is usually less demanding in terms of circuit power than transmission. Finally, in sleep mode, a node waits to transmit/receive and does not perform any processing, such that only a fraction of the circuit power is consumed [4], i.e. $P^{\mathrm{Sl}}=n \cdot P^{\mathrm{SlpA}}$, where $P^{\mathrm{SlpA}}$ is the perantenna sleep power. By inserting the definitions of $P^{\mathrm{Tx}}$ in (3), $P^{\mathrm{Rx}}$ in (4) and $P^{\mathrm{Id}}$ into (2), the total consumed power of the cooperative MIMO-AF system in Fig. 1 is reformulated as

$$
P_{\Sigma}=P_{c}+\Delta_{\mathrm{SN}} P_{\mathrm{SN}}+\Delta_{\mathrm{RN}} P_{\mathrm{RN}}
$$

where, as in [14],

$$
\begin{aligned}
P_{\mathrm{SN}}(\mathbf{R}) & =\operatorname{tr}\left\{\mathbf{R} \mathbf{R}^{\dagger}\right\} \text { and } \\
P_{\mathrm{RN}}(\mathbf{R}, \mathbf{G}) & =\operatorname{tr}\left\{\mathbf{G}\left(\sigma_{1}^{2} \mathbf{I}_{n_{\mathrm{RN}}}+\mathbf{H}_{1} \mathbf{R} \mathbf{R}^{\dagger} \mathbf{H}_{1}^{\dagger}\right) \mathbf{G}^{\dagger}\right\} .
\end{aligned}
$$

In addition, $P_{c}=n_{\mathrm{SN}}\left(P_{\mathrm{SN}}^{\mathrm{CipA}}+P_{\mathrm{SN}}^{\mathrm{SlpA}}\right)+P_{\mathrm{SN}}^{\mathrm{Ci}}+(1+$ $\varsigma)\left(n_{\mathrm{RN}} P_{\mathrm{RN}}^{\mathrm{CipA}}+P_{\mathrm{RN}}^{\mathrm{Ci}}\right)+2 \varsigma\left(n_{\mathrm{DN}} P_{\mathrm{DN}}^{\mathrm{CipA}}+P_{\mathrm{DN}}^{\mathrm{Ci}}\right)$ accounts for all the fixed circuit consumed powers. Hence, based on (5) and (6), $P_{\Sigma}$ can be expressed in two distinct manners as

$$
\begin{aligned}
& P_{\Sigma}(\mathbf{R}, \mathbf{G})=P_{c}^{\prime}(\mathbf{R})+\frac{\sigma_{1}^{2}}{\sigma_{2}^{2}} \operatorname{tr}\left\{\mathbf{G} \ddot{\boldsymbol{\Upsilon}}(\mathbf{R}) \mathbf{G}^{\dagger}\right\} \text { or } \\
& P_{\Sigma}(\mathbf{R}, \mathbf{G})=P_{c}^{\prime}(\mathbf{G})+\operatorname{tr}\left\{\mathbf{R}^{\dagger} \ddot{\mathbf{\Psi}}\left(\Delta_{\mathrm{SN}}, \Delta_{\mathrm{RN}}, \mathbf{G}\right) \mathbf{R}\right\},
\end{aligned}
$$

where

$$
\begin{aligned}
P_{c}^{\prime}(\mathbf{R}) & =P_{c}+\Delta_{\mathrm{SN}} P_{\mathrm{SN}}(\mathbf{R}), \\
P_{c}^{\prime}(\mathbf{G}) & =P_{c}+\Delta_{\mathrm{RN}} \sigma_{1}^{2} \operatorname{tr}\left\{\mathbf{G G}^{\dagger}\right\}, \\
\ddot{\mathbf{\Upsilon}}(\mathbf{R}) & =\frac{\sigma_{2}^{2}}{\sigma_{1}^{2}} \Delta_{\mathrm{RN}}\left(\sigma_{1}^{2} \mathbf{I}_{n_{\mathrm{RN}}}+\mathbf{H}_{1} \mathbf{R R}^{\dagger} \mathbf{H}_{1}^{\dagger}\right), \text { and } \\
\ddot{\mathbf{\Psi}}(\alpha, \beta, \mathbf{G}) & =\alpha \mathbf{I}_{n_{\mathrm{SN}}}+\beta \mathbf{H}_{1}^{\dagger} \mathbf{G}^{\dagger} \mathbf{G} \mathbf{H}_{1} .
\end{aligned}
$$

The energy consumption, $E_{b}$, (or EE, $1 / E_{b}$ ) being simply a ratio between consumed power and rate [29], the EE-SE tradeoff of the cooperative MIMO-AF system in Fig. 1 can be expressed as

$$
E_{b}(\mathbf{R}, \mathbf{G})=\frac{P_{\Sigma}(\mathbf{R}, \mathbf{G})}{R_{\Sigma}(\mathbf{R}, \mathbf{G})},
$$

where detailed expressions for $R_{\Sigma}(\mathbf{R}, \mathbf{G})$ and $P_{\Sigma}(\mathbf{R}, \mathbf{G})$ are provided in (1) and (7), respectively.

\section{EE-Optimal Source And Relay Precoding}

In this section, our aim is to derive precoding matrices $\mathbf{R}$ and $\mathbf{G}$ that minimizes the following optimization problem

$$
\begin{array}{ll}
\min _{\mathbf{R}, \mathbf{G}} & E_{b}(\mathbf{R}, \mathbf{G}), \\
\text { s.t. } & P_{\mathrm{SN}}(\mathbf{R}) \leq P_{\mathrm{SN}}^{\max }, \\
& P_{\mathrm{RN}}(\mathbf{R}, \mathbf{G}) \leq P_{\mathrm{RN}}^{\max },
\end{array}
$$

where $\mathbf{R} \succeq \mathbf{0}, \mathbf{R} \neq \mathbf{0}$, and $\mathbf{G} \succeq \mathbf{0}$. In addition, $P_{\mathrm{SN}}^{\max }$ and $P_{\mathrm{RN}}^{\max }$ are the maximum transmit power at the $\mathrm{SN}$ and $\mathrm{RN}$, respectively. Contrary to sum-rate maximization (e.g. [16], [17]), or transmit power and MSE minimization problems (e.g. [20], [22]), the EE objective function in (9a) is not a Schur-concave/convex objective function, but a ratio between Schur-concave/convex functions. As such, contrary to Schurconcave/convex objective functions, (9a) exhibits a global optimum (which is not 0 or $\infty$ as long as $P_{c}>0$ [30]) even when no constraints are enforced. Indeed, as it explained in [31], the sole EE-optimal solution for a given EE optimization problem can only be obtained by solving its unconstrained form [31], i.e. solving (9a) without (9b) and (9c). Consequently, in order to optimally solve the optimization problem at hand, it is necessary to first solve (9a) on its own and refine its solution if it does not meet the constraints in (9b) and (9c), as it is further detailed in Sections III-A and III-B.

The problem in (9) is generally non-convex, since (9a) is not necessarily jointly convex in $\mathbf{R}$ and $\mathbf{G}$. However, it can be proved, by relying on Propositions 1, 2 and 3, that this optimization problem has a unique global minimum when $\mathbf{R}$ and $\mathbf{G}$ are treated independently. In other words, there exists an optimal matrix $\mathbf{R}^{\star}$ minimizing (9) for a known $\mathbf{G}$ and an optimal matrix $\mathbf{G}^{\star}$ minimizing (9) for a known $\mathbf{R}$.

Proposition 1: Functions of the type

$$
f(\mathbf{X})=p(\mathbf{X}) / q(\mathbf{X}),
$$

where $p$ is a linear function of $\mathbf{X}$ and $q$ is a strictly concave function of $\mathbf{X}$, are strictly pseudo-convex functions of $\mathbf{X}$, for $\mathbf{X} \succeq \mathbf{0}$. See section A of the Appendix for the proof. In turn, according to [32], if $\mathbf{X}^{\star}$ is a stationary point of $f$, i.e. a point where $\nabla_{\mathbf{X}} f\left(\mathbf{X}=\mathbf{X}^{\star}\right)=\mathbf{0}$, then $f^{\star}=f\left(\mathbf{X}^{\star}\right)$ is the unique global minimum of $f$ over its domain.

Proposition 2: Functions of the type

$$
f(\mathbf{X})=p(\mathbf{X})+q(\mathbf{X}),
$$

where $p$ is a linear function of $\mathbf{X}$ and $q$ is a strictly pseudoconvex function of $\mathbf{X}$, are strictly pseudo-convex functions of $\mathbf{X}$, for $\mathbf{X} \succeq \mathbf{0}$. See section B of the Appendix for the proof.

Proposition 3: Functions of the type

$$
f(\mathbf{X})=a / q(\mathbf{X}),
$$

where $a$ is a constant and $q$ is a strictly positive and concave function of $\mathbf{X}$, are convex functions of $\mathbf{X}$, for $\mathbf{X} \succeq \mathbf{0}$.

Proof: Based on the scalar composition rules in [33], we know that a function $f=h \circ g$ is convex if $h$ is convex and non-increasing, and $g$ is concave. Given that $h(x)=\frac{a}{x}$ is convex and non-increasing function of $x$ for $x>0$, it implies that $f$ would be convex if $g>0$ is concave. 
In the following, we first derive $\mathbf{R}^{\star}$ for a known $\mathbf{G}$ and then $\mathbf{G}^{\star}$ for a known $\mathbf{R}$. Next, an alternating optimization approach is utilized (as in [10], [16], [20]) to find a global solution to the problem in (9), as it is further detailed in Section III-C.

\section{A. EE-Optimal Source Precoding}

Based on equations (9), (8), (7b) and (1b), finding the EE-optimal source precoding matrix, $\mathbf{R}^{\star}$, for a given relay precoding matrix $\mathbf{G}$ boils down to solving $\mathbf{R}^{\star}=$

$\arg \min _{\mathbf{R}} E_{b}(\mathbf{R}, \mathbf{G})=\frac{P_{c}^{\prime}(\mathbf{G})+\operatorname{tr}\left\{\mathbf{R}^{\dagger} \ddot{\mathbf{\Psi}}\left(\Delta_{\mathrm{SN}}, \Delta_{\mathrm{RN}}, \mathbf{G}\right) \mathbf{R}\right\}}{W \log _{2}\left|\mathbf{I}_{n_{\mathrm{SN}}}+\mathbf{R}^{\dagger} \dot{\Psi}(\mathbf{G}) \mathbf{R}\right|}$,

subject to (9b) and (9c). Given that $\operatorname{tr}\{\mathbf{A B}\}=\operatorname{tr}\{\mathbf{B A}\}$ and $|\mathbf{I}+\mathbf{A B}|=|\mathbf{I}+\mathbf{B A}|$ (Sylvester's determinant identity) for any matrices $\mathbf{A}$ and $\mathbf{B}, E_{b}(\mathbf{R}, \mathbf{G})$ in (13) is equivalent to

$$
E_{b}(\mathbf{R})=\frac{P_{c}^{\prime}+\operatorname{tr}\left\{\mathbf{R R}^{\dagger} \ddot{\Psi}\left(\Delta_{\mathrm{SN}}, \Delta_{\mathrm{RN}}\right)\right\}}{W \log _{2}\left|\mathbf{I}_{n_{\mathrm{SN}}}+\dot{\Psi}^{\frac{1}{2}} \mathbf{R} \mathbf{R}^{\dagger} \dot{\Psi}^{\frac{1}{2}}\right|}
$$

since $\dot{\Psi} \succ 0$. Note that the argument $\mathbf{G}$ is omitted in (14) and in the rest of sub-Section III-A for simplifying the notation. By applying the change of variables $\mathbf{Y}=\dot{\boldsymbol{\Psi}}^{\frac{1}{2}} \mathbf{R} \mathbf{R}^{\dagger} \dot{\boldsymbol{\Psi}}^{\frac{1}{2}}$ (such that $\mathbf{Y} \succeq \mathbf{0}, \mathbf{Y} \neq \mathbf{0}$, is a Hermitian matrix) and $\boldsymbol{\Psi}(\alpha, \beta)=\left(\dot{\boldsymbol{\Psi}}^{-1}\right)^{\frac{1}{2}} \ddot{\boldsymbol{\Psi}}(\alpha, \beta)\left(\dot{\boldsymbol{\Psi}}^{-1}\right)^{\frac{1}{2}}$, to (14), (9b) and (9c), the optimization problem in (13) can be re-expressed as

$$
\begin{aligned}
\mathbf{Y}^{\star}= & \arg \min _{\mathbf{Y}} E_{b}(\mathbf{Y})=\frac{P_{c}^{\prime}+\Delta_{\mathrm{SN}} P_{\mathrm{SN}}(\mathbf{Y})+\Delta_{\mathrm{RN}} \bar{P}_{\mathrm{RN}}(\mathbf{Y})}{R_{\Sigma}(\mathbf{Y})}, \\
& \text { s.t. } P_{\mathrm{SN}}(\mathbf{Y}) \leq P_{\mathrm{SN}}^{\max } \text { and } \bar{P}_{\mathrm{RN}}(\mathbf{Y}) \leq \bar{P}_{\mathrm{RN}}^{\max },
\end{aligned}
$$

where

$$
\begin{aligned}
R_{\Sigma}(\mathbf{Y}) & =W \log _{2}\left|\mathbf{I}_{n_{\mathrm{SN}}}+\mathbf{Y}\right|, \\
P_{\mathrm{SN}}(\mathbf{Y}) & =\operatorname{tr}\{\mathbf{Y} \mathbf{\Psi}(1,0)\}, \text { and } \\
\bar{P}_{\mathrm{RN}}(\mathbf{Y}) & =\operatorname{tr}\{\mathbf{Y} \mathbf{\Psi}(0,1)\} .
\end{aligned}
$$

In addition, $\bar{P}_{\mathrm{RN}}^{\max }=\left(P_{\mathrm{RN}}^{\max }-\sigma_{1}^{2} \operatorname{tr}\left\{\mathbf{G G}^{\dagger}\right\}\right)$. In turn, the Lagrangian associated to (16) can expressed as $\mathcal{L}\left(\mathbf{Y}, \lambda_{1}, \lambda_{2}\right)=$

$$
\left\{\begin{array}{l}
E_{b}(\mathbf{Y})=\frac{P_{c}^{\prime}+\operatorname{tr}\left\{\mathbf{Y} \mathbf{\Psi}\left(\Delta_{\mathrm{SN}}, \Delta_{\mathrm{RN}}\right)\right\}}{R_{\Sigma}(\mathbf{Y})}, \\
\frac{P_{c}^{\prime}+\Delta_{\mathrm{SN}} P_{\mathrm{SN}}^{\max }+\Delta_{\mathrm{RN}} \bar{P}_{\mathrm{RN}}(\mathbf{Y})}{R_{\Sigma}(\mathbf{Y})}+\lambda_{1}\left(P_{\mathrm{SN}}(\mathbf{Y})-P_{\mathrm{SN}}^{\max }\right), \\
\frac{P_{c}^{\prime}+\Delta_{\mathrm{SN}} P_{\mathrm{SN}}(\mathbf{Y})+\Delta_{\mathrm{RN}} \bar{P}_{\mathrm{RN}}^{\max }}{R_{\Sigma}(\mathbf{Y})}+\lambda_{2}\left(\bar{P}_{\mathrm{RN}}(\mathbf{Y})-\bar{P}_{\mathrm{RN}}^{\max }\right), \text { or } \\
\frac{P_{c}^{\prime}+\Delta_{\mathrm{SN}} P_{\mathrm{SN}}^{\max }+\Delta_{\mathrm{RN}} \bar{P}_{\mathrm{RN}}^{\max }}{R_{\Sigma}(\mathbf{Y})}+\lambda_{1}\left(P_{\mathrm{SN}}(\mathbf{Y})-P_{\mathrm{SN}}^{\max }\right) \\
+\lambda_{2}\left(\bar{P}_{\mathrm{RN}}(\mathbf{Y})-\bar{P}_{\mathrm{RN}}^{\max }\right),
\end{array}\right.
$$

in the unconstrained case, i.e. if $P_{\mathrm{SN}}\left(\mathbf{Y}^{\star}\right)<P_{\mathrm{SN}}^{\max }$ and $\bar{P}_{\mathrm{RN}}\left(\mathrm{Y}^{\star}\right)<\bar{P}_{\mathrm{RN}}^{\max }$, single power constrained (SPC) case at the SN, i.e. if $P_{\mathrm{SN}}\left(\mathbf{Y}^{\star}\right) \geq P_{\mathrm{SN}}^{\max }$ and $\bar{P}_{\mathrm{RN}}\left(\mathbf{Y}^{\star}\right)<\bar{P}_{\mathrm{RN}}^{\max }$, SPC case at the RN, i.e. if $P_{\mathrm{SN}}\left(\mathbf{Y}^{\star}\right)<P_{\mathrm{SN}}^{\max }$ and $\bar{P}_{\mathrm{RN}}\left(\mathbf{Y}^{\star}\right) \geq \bar{P}_{\mathrm{RN}}^{\max }$, or dual power constrained (DPC) case, i.e. if $P_{\mathrm{SN}}\left(\mathbf{Y}^{\star}\right) \geq P_{\mathrm{SN}}^{\max }$ and $\bar{P}_{\mathrm{RN}}\left(\mathbf{Y}^{\star}\right) \geq \bar{P}_{\mathrm{RN}}^{\max }$, respectively. In addition, $\lambda_{1} \geq 0$ and $\lambda_{2} \geq 0$ are Lagrange multipliers.
Corollary 1: The Lagrangian function in (17) is a strictly pseudo-convex (in the unconstrained and both SPC cases) or a convex function (in the DPC case) of $\mathbf{Y}$, such that it has a unique global minimum, $E_{b}^{\star}=E_{b}\left(\mathbf{Y}^{\star}\right)=E_{b}\left(\mathbf{R}^{\star}\right)$.

Proof: Given that both $P_{\mathrm{SN}}(\mathbf{Y})$ and $\bar{P}_{\mathrm{RN}}(\mathbf{Y})$ are linear functions of $\mathbf{Y}$ and $R_{\Sigma}(\mathbf{Y})$ is a strictly positive and concave function of $\mathbf{Y}$, as it is fully proved in section $\mathrm{C}$ of the Appendix, the Lagrangian function in (17) meets the requirements of Propositions 1 (unconstrained and SPC cases), 2 (SPC cases) and 3 (DPC case).

Proposition 4: The optimal source precoding matrix, $\mathbf{R}^{\star}$, i.e. the optimal solution to the optimization problem in (13) can be expressed in closed-form as

$$
\mathbf{R}^{\star}=\left[\left(\dot{\Psi}^{-1}\right)^{\frac{1}{2}} \mathbf{U}_{\Psi} \widehat{\mathbf{Y}}^{\star} \mathbf{U}_{\Psi}^{\dagger}\left(\dot{\Psi}^{-1}\right)^{\frac{1}{2}}\right]^{\frac{1}{2}},
$$

where $\mathbf{U}_{\Psi}$ is a unitary matrix that contains the eigenvectors of $\boldsymbol{\Psi}(\alpha, \beta)$. In addition, $\widehat{\mathbf{Y}}^{\star}=\operatorname{diag}\left(\left[y_{1}^{\star}, y_{2}^{\star}, \ldots, y_{n_{\mathrm{SN}}}^{\star}\right]\right)$ is a diagonal matrix with diagonal elements such as

$$
y_{i}^{\star}=\left[\frac{W E_{b}^{\star}}{\ln (2)} \frac{1}{\psi_{i}(\alpha, \beta)}-1\right]_{+}, \forall i \in\left\{1, \ldots, n_{\mathrm{SN}}\right\},
$$

where $\psi_{i}(\alpha, \beta)$ are the eigenvalues of $\boldsymbol{\Psi}(\alpha, \beta)$. See section $\mathrm{D}$ of the Appendix for the proof. In addition, by inserting the optimal precoding structure, $\mathbf{R}^{\star}$ in (18), into (14), arguments of both the trace and determinant operators become diagonal matrices such that (14) simplifies as

$$
E_{b}^{\star}=\frac{P_{c}^{\prime}+\sum_{i=1}^{n_{\mathrm{SN}}} y_{i}^{\star} \psi_{i}(\alpha, \beta)}{W \sum_{i=1}^{n_{\mathrm{SN}}} \log _{2}\left(1+y_{i}^{\star}\right)} .
$$

The values of $\alpha$ and $\beta$ in (19) as well as (20) depend on how constrained the problem in (13) or (15) is.

1) Unconstrained Optimization: In this case, $\alpha=\Delta_{\mathrm{SN}}$ and $\beta=\Delta_{\mathrm{RN}}$ in both (19) and (20) (see Section D of the Appendix for more details). Consequently, the problem of finding $\mathbf{R}^{\star}$ boils down to finding $E_{b}^{\star}$ that satisfies equation (20), i.e. a univariate root-search problem since (20) is expressed solely as a function $E_{b}^{\star}$ (via $y_{i}^{\star}$ ). This problem can be solved in a lowcomplexity manner by using a classic univariate root-finding algorithm, as it is detailed in Algorithm 1.

2) Single Power Constrained Optimization: In the SPC case at the SN, $\alpha=\lambda_{1}^{\star}$ and $\beta=\Delta_{\mathrm{RN}}$ in both (19) and (20); whereas in the SPC case at the RN, $\alpha=\Delta_{\mathrm{SN}}$ and $\beta=\lambda_{2}^{\star}$ in both (19) and (20), where $\lambda_{1}^{\star}$ and $\lambda_{2}^{\star}$ are variables to optimize (see Section D of the Appendix for more details). Thus, in the SPC case at the SN, the problem of finding $\mathbf{R}^{\star}$ is equivalent to finding the variable $\lambda_{1}^{\star}$ that minimizes $E_{b}^{\star}$ in (20) when the following power constraint equation (obtained by inserting (18) into (9b)) is satisfied

$$
\sum_{i=1}^{n_{\mathrm{SN}}} y_{i}^{\star} \check{\psi}_{i}(1,0)=P_{\mathrm{SN}}^{\max }
$$

where $\check{\psi}_{i}(1,0)$ are the diagonal elements of $\mathbf{U}_{\Psi}^{\dagger} \Psi(1,0) \mathbf{U}_{\Psi}$. Similarly, in the SPC case at the RN, it is required to find $\lambda_{2}^{\star}$ that minimizes $E_{b}^{\star}$ in (20) when

$$
\sum_{i=1}^{n_{\mathrm{SN}}} y_{i}^{\star} \check{\psi}_{i}(0,1)=\bar{P}_{\mathrm{RN}}^{\max }
$$




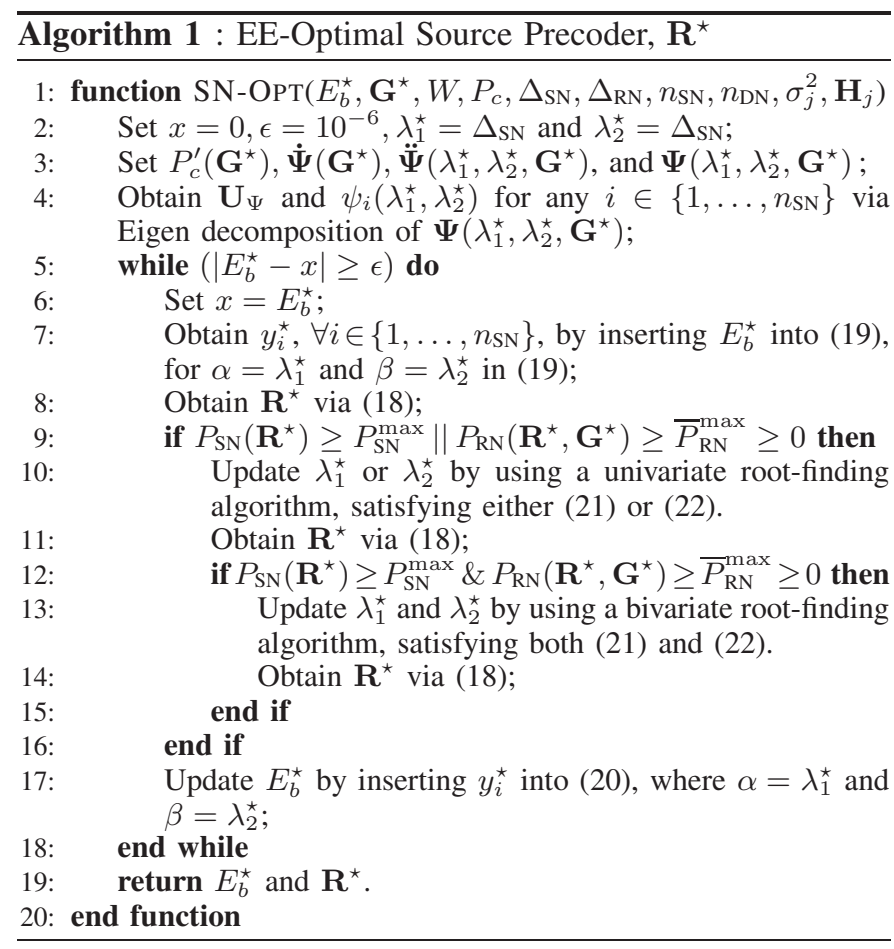

is satisfied (obtained by inserting (18) into (9c)), where $\check{\psi}_{i}(0,1)$ are the diagonal elements of $\mathbf{U}_{\Psi}^{\dagger} \boldsymbol{\Psi}(0,1) \mathbf{U}_{\Psi}$. Finding $E_{b}^{\star}$ for a fixed $\lambda_{i}^{\star}, i=1$ or 2 , via (20) can be computed as in the unconstrained case, while finding $\lambda_{i}^{\star}$ for a fixed $E_{b}^{\star}$ via (21) or (22) is a univariate root-finding problem, which can be solved in a low-complexity manner by using a classic root-finding algorithm (e.g. Newton-Raphson method [34]).

3) Dual Power Constrained Optimization: In this case $\alpha=\lambda_{1}^{\star}$ and $\beta=\lambda_{2}^{\star}$ in both (19) and (20) (see Section D of the Appendix for more details). Consequently, the problem of finding $\mathbf{R}^{\star}$ boils down to finding the variables $\lambda_{1}^{\star}$ and $\lambda_{2}^{\star}$ that minimizes $E_{b}^{\star}$ in (20) when both power constraint equations in (21) and (22) are satisfied.

The different steps to obtain the EE-optimal source matrix $\mathbf{R}^{\star}$ for a known $\mathbf{G}$ are summarized in Algorithm 1 .

\section{B. EE-optimal Relay Precoding}

Based on equations (9), (8), (7a) and (1a), finding the EE-optimal relay precoding matrix, $\mathbf{G}^{\star}$, for a given relay precoding matrix $\mathbf{R}$ boils down to solving $\mathbf{G}^{\star}=$ $\arg \min _{\mathbf{G}} E_{b}(\mathbf{R}, \mathbf{G})=$

$$
\frac{P_{c}^{\prime}(\mathbf{R})+\frac{\sigma_{1}^{2}}{\sigma_{2}^{2}} \operatorname{tr}\left\{\mathbf{G} \ddot{\mathbf{\Upsilon}}(\mathbf{R}) \mathbf{G}^{\dagger}\right\}}{R_{0}(\mathbf{R})+W \log _{2}\left|\boldsymbol{\Omega}\left(\mathbf{G} \boldsymbol{\Upsilon}(\mathbf{R})^{1 / 2}\right) \boldsymbol{\Omega}(\mathbf{G})^{-1}\right|},
$$

subject to (9c), where $\mathbf{\Upsilon}(\mathbf{R})=\mathbf{I}_{n_{\mathrm{RN}}}+\dot{\boldsymbol{\Upsilon}}(\mathbf{R})$. In turn, the Lagrangian associated to (23) can expressed as

$$
\mathcal{L}(\mathbf{G}, \mu)=\left\{\begin{array}{l}
E_{b}(\mathbf{G}) \text { or } \\
\frac{P_{c}^{\prime}+\Delta_{\mathrm{RN}} P_{\mathrm{RN}}^{\max }}{R_{\Sigma}(\mathbf{G})}+\mu\left(P_{\mathrm{RN}}(\mathbf{G})-P_{\mathrm{RN}}^{\max }\right),
\end{array}\right.
$$

in the unconstrained or power constrained case, i.e. if $P_{\mathrm{RN}}\left(\mathbf{G}^{\star}\right)<P_{\mathrm{RN}}^{\max }$ or $P_{\mathrm{RN}}\left(\mathbf{G}^{\star}\right) \geq P_{\mathrm{RN}}^{\max }$ in $(9 \mathrm{c})$, respectively, where $\mu \geq 0$ is a Lagrange multiplier and $R_{\Sigma}(\mathbf{G})$ is given in (1a). Note that the argument $\mathbf{R}$ is omitted in (24) and in the rest of sub-Section III-B for simplifying the notation. By applying the change of variables $\mathbf{Z}=\frac{\sigma_{1}^{2}}{\sigma_{2}^{2}} \mathbf{G}^{\dagger} \mathbf{H}_{2}^{\dagger} \mathbf{H}_{2} \mathbf{G}$ (such that $\mathbf{Z} \succeq \mathbf{0}$ is a Hermitian matrix) to the Lagrangian function in (24), the latter can be re-expressed as

$$
\mathcal{L}(\mathbf{Z}, \mathbf{W}, \mu)=\left\{\begin{array}{l}
E_{b}(\mathbf{Z}, \mathbf{W})=\frac{P_{c}^{\prime}+\Delta_{\mathrm{RN}} P_{\mathrm{RN}}(\mathbf{Z}, \mathbf{W})}{R_{\Sigma}(\mathbf{Z})} \text { or } \\
\frac{P_{c}^{\prime}+\Delta_{\mathrm{RN}} P_{\mathrm{RN}}^{\max }}{R_{\Sigma}(\mathbf{Z})}+\mu\left(P_{\mathrm{RN}}(\mathbf{Z}, \mathbf{W})-P_{\mathrm{RN}}^{\max }\right),
\end{array}\right.
$$

where

$$
\begin{gathered}
R_{\Sigma}(\mathbf{Z})=R_{0}+W \log _{2}\left|\frac{\mathbf{I}_{n_{\mathrm{RN}}}+\mathbf{\Upsilon}^{1 / 2} \mathbf{Z} \mathbf{\Upsilon}^{1 / 2}}{\mathbf{I}_{n_{\mathrm{RN}}}+\mathbf{Z}}\right| \text { and } \\
P_{\mathrm{RN}}(\mathbf{Z}, \mathbf{W})=\frac{1}{\Delta_{\mathrm{RN}}} \operatorname{tr}\left\{\mathbf{Z}^{1 / 2} \mathbf{W}\left(\mathbf{H}_{2} \mathbf{H}_{2}^{\dagger}\right)^{-1} \mathbf{W}^{\dagger} \mathbf{Z}^{1 / 2} \ddot{\mathbf{\Upsilon}}\right\}
\end{gathered}
$$

Moreover, $\mathbf{W} \in \mathbb{C}^{n_{\mathrm{RN}} \times n_{\mathrm{DN}}}, \mathbf{W} \mathbf{W}^{\dagger}=\mathbf{I}_{n_{\mathrm{RN}}}$ (if $n_{\mathrm{DN}} \geq n_{\mathrm{RN}}$ ) in (26). Contrary to (15), it is not possible to easily solve (23) and obtain its global minimum $E_{b}^{\star}=E_{b}\left(\mathbf{G}^{\star}\right)$; even though $R_{\Sigma}(\mathbf{Z})$ is a strictly positive and concave function of $\mathbf{Z}$, as it is proved in section $\mathrm{E}$ of the Appendix, $P_{\Sigma}(\mathbf{Z}, \mathbf{W})$ is not a linear function of $\mathbf{Z}$ and, hence, the convexity of (24) or (25) cannot be readily established. Nevertheless, it can be proved, as it is further explained in the following, that (25) can be lower and upper bounded by strictly pseudo-convex or convex functions (i.e. meeting the requirements of Propositions 1 and $3)$. In turn, we prove that $E_{b}^{-} \leq E_{b}^{\star} \leq E_{b}^{+}$, where $E_{b}^{-}$and $E_{b}^{+}$are the global minima of the lower and upper bounds of (25), correspondingly. Based on these bounds, two methods are then proposed to solve (23); an EE-optimal method and a low-complexity energy-efficient (but suboptimal) method.

1) Lower and Upper Bounds of (25): Let $\mathbf{G}$ be decomposed as

$$
\mathbf{G}=\mathbf{V}_{\mathbf{G}} \widehat{\mathbf{G}}^{\frac{1}{2}} \mathbf{U}_{\mathbf{G}}^{\dagger},
$$

where $\mathbf{V}_{\mathbf{G}}$ as well as $\mathbf{U}_{\mathbf{G}}^{\dagger}$ are unitary matrices and $\widehat{\mathbf{G}}$ is a diagonal matrix containing the eigenvalues of $\mathbf{G}^{\dagger} \mathbf{G}$ sorted in descending order, such that $\mathbf{G}^{\dagger} \mathbf{G}=\mathbf{U}_{\mathbf{G}} \widehat{\mathbf{G}} \mathbf{U}_{\mathbf{G}}^{\dagger}$. For instance in [10], [11], [14], [25], $\mathbf{V}_{\mathbf{G}}=\mathbf{V}_{2}$ and $\mathbf{U}_{\mathbf{G}}=\mathbf{U}_{1}$, where $\mathbf{V}_{2}$ contains the right-singular vectors of $\mathbf{H}_{2}$ and $\mathbf{U}_{1}$ contains the left-singular vectors of $\mathbf{H}_{1}$. In addition, let $\widehat{\Lambda}$ be a diagonal matrix containing the eigenvalues of $\mathbf{H}_{2}^{\dagger} \mathbf{H}_{2}$ sorted in descending order, such that $\mathbf{H}_{2}^{\dagger} \mathbf{H}_{2}=\mathbf{V}_{2} \widehat{\Lambda} \mathbf{V}_{2}^{\dagger}$.

a) Lower Bound:

Proposition 5: The function $P_{\mathrm{RN}}(\mathbf{Z}, \mathbf{W})$ in $(26 \mathrm{~b})$ can be lower bounded by

$$
P_{\mathrm{RN}}(\widehat{\mathbf{Z}})=\frac{1}{\Delta_{\mathrm{RN}}} \operatorname{tr}\left\{\widehat{\mathbf{Z}} \widehat{\boldsymbol{\Lambda}}^{-1} \widehat{\ddot{\boldsymbol{\Upsilon}}}^{\uparrow}\right\},
$$

i.e. $P_{\mathrm{RN}}(\widehat{\mathbf{Z}}) \leq P_{\mathrm{RN}}(\mathbf{Z}, \mathbf{W})$, where $\widehat{\mathbf{Z}}=\frac{\sigma_{1}^{2}}{\sigma_{2}^{2}} \widehat{\mathbf{G}} \widehat{\mathbf{\Lambda}}$ is a diagonal matrix and $\widehat{\ddot{\Upsilon}}^{\uparrow}$ is a diagonal matrix containing the eigenvalues of $\ddot{\Upsilon}$ sorted in ascending order. Whereas the function $R_{\Sigma}(\mathbf{Z})$ in (26a) can be upper bounded by

$$
R_{\Sigma}(\widehat{\mathbf{Z}})=R_{0}+W \log _{2}\left|\mathbf{I}_{n_{\mathrm{RN}}}+\widehat{\mathbf{Z}} \widehat{\Upsilon}\right|-W \log _{2}\left|\mathbf{I}_{n_{\mathrm{RN}}}+\widehat{\mathbf{Z}}\right|,
$$


i.e. $1 / R_{\Sigma}(\widehat{\mathbf{Z}}) \leq 1 / R_{\Sigma}(\mathbf{Z})$, where $\widehat{\boldsymbol{\Upsilon}}$ is a diagonal matrix containing the eigenvalues of $\Upsilon$ sorted in descending order,

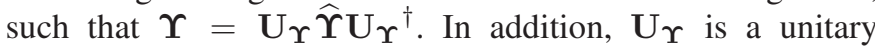
matrix containing the eigenvectors of $\Upsilon$. See section $F$ of the Appendix for the proof. Thus, the following Lagrangian function

$$
\mathcal{L}(\widehat{\mathbf{Z}}, \mu)=\left\{\begin{array}{l}
E_{b}(\widehat{\mathbf{Z}})=\frac{P_{c}^{\prime}+\Delta_{\mathrm{RN}} P_{\mathrm{RN}}(\widehat{\mathbf{Z}})}{R_{\Sigma}(\widehat{\mathbf{Z}})} \text { or } \\
\frac{P_{c}^{\prime}+\Delta_{\mathrm{RN}} P_{\mathrm{RN}}^{\max }}{R_{\Sigma}(\widehat{\mathbf{Z}})}+\mu\left(P_{\mathrm{RN}}(\widehat{\mathbf{Z}})-P_{\mathrm{RN}}^{\max }\right),
\end{array}\right.
$$

is a lower bound of the Lagrangian of the original problem in (24) or (25).

Corollary 2: Contrary to (25), the Lagrangian function in (30) is a strictly pseudo-convex (unconstrained case) or convex function (power constrained case) of $\widehat{\mathbf{Z}}$, respectively, such that it has a unique global minimum.

Proof: Given that $P_{\mathrm{RN}}(\widehat{\mathbf{Z}})$ is a linear function of $\widehat{\mathbf{Z}}$ and $R_{\Sigma}(\widehat{\mathbf{Z}})$ is a strictly positive and concave function of $\widehat{\mathbf{Z}}$ (same proof as for $R_{\Sigma}(\mathbf{Z})$ in Section $\mathrm{E}$ of the Appendix), the Lagrangian function in (30) meets the requirements of Propositions 1 and 3.

Corollary 3: Let $E_{b}^{-}$be the global minimum of (30), it then implies that $E_{b}^{-} \leq E_{b}^{\star}$, (given that $\mathcal{L}(\widehat{\mathbf{Z}}, \mu) \leq \mathcal{L}(\mathbf{Z}, \mathbf{W}, \mu)$ ).

\section{b) Upper Bound:}

Proposition 6: Let $\mathrm{G}^{+}$be the relay precoding matrix that minimizes (23) subject to (9c) and $\mathbf{G}=\mathbf{V}_{2} \widehat{\mathbf{G}}^{\frac{1}{2}} \mathbf{U}_{\Upsilon}^{\dagger}$, i.e.

$$
\begin{aligned}
E_{b}^{+}=\min _{\widehat{\mathbf{G}}, \mathbf{V}_{\mathbf{G}}, \mathbf{U}_{\mathbf{G}}} & E_{b}\left(\widehat{\mathbf{G}}, \mathbf{V}_{\mathbf{G}}, \mathbf{U}_{\mathbf{G}}\right) \\
\text { s.t. } & (9 \mathrm{c}), \mathbf{V}_{\mathbf{G}}=\mathbf{V}_{2}, \text { and } \mathbf{U}_{\mathbf{G}}=\mathbf{U}_{\Upsilon} ;
\end{aligned}
$$

it then implies that the global minimum of $(31), E_{b}^{+}=$ $E_{b}\left(\mathbf{G}^{+}\right)$, verifies $E_{b}^{+} \geq E_{b}^{\star}$.

Proof: It is commonly known in optimization theory [33] that enforcing extra constraints to an optimization problem results in a reduction of the set of possible/feasible solutions for the given problem; in other words, the new solution space becomes a subset of the original solution space. Consequently, it implies that the solution of a minimization problem is always lower or equal to the solution of the same problem when extra constraints are enforced on it; in other words, the solution of the problem with extra constraints upper bounds the solution of the original problem. Based on this premise, it can be straightforwardly concluded that

$$
\begin{aligned}
E_{b}^{\star}=\min _{\mathbf{G}} & E_{b}(\mathbf{G})=\min _{\widehat{\mathbf{G}}, \mathbf{V}_{\mathbf{G}}, \mathbf{U}_{\mathbf{G}}} E_{b}\left(\widehat{\mathbf{G}}, \mathbf{V}_{\mathbf{G}}, \mathbf{U}_{\mathbf{G}}\right) \leq(31) \\
\text { s.t. } \quad(9 \mathrm{c}), & \text { s.t. } \quad(9 \mathrm{c}) .
\end{aligned}
$$

Indeed, the solution space in (31) is restricted to the domain of positive semi-definite matrix having a specific structure, i.e. a structure as in (27) with $\mathbf{V}_{\mathbf{G}}=\mathbf{V}_{2}$ and $\mathbf{U}_{\mathbf{G}}=\mathbf{U}_{\Upsilon}$, instead of the whole positive semi-definite matrix domain (as in the left side of the inequality).

Corollary 4: Contrary to (23), the optimization problem in (31) is strictly-pseudo convex or convex in the unconstrained or power constrained case, respectively. Indeed, its Lagrangian can be expressed as in (30) with $R_{\Sigma}(\widehat{\mathbf{Z}})$ expressed as in (29) and $P_{\mathrm{RN}}(\widehat{\mathbf{Z}})$ given by

$$
P_{\mathrm{RN}}(\widehat{\mathbf{Z}})=\frac{1}{\Delta_{\mathrm{RN}}} \operatorname{tr}\left\{\widehat{\mathbf{Z}} \widehat{\boldsymbol{\Lambda}}^{-1} \overline{\ddot{\mathbf{\Upsilon}}}\right\},
$$

instead of (28), where $\overline{\ddot{\Upsilon}}=\mathbf{U}_{\Upsilon}{ }^{\dagger} \ddot{\Upsilon} \mathbf{U}_{\Upsilon} \succeq \mathbf{0}$ is a Hermitian matrix. Note that $P_{\mathrm{RN}}(\widehat{\mathbf{Z}})$ in (32) is a linear function of $\widehat{\mathbf{Z}}$.

Proof: Same as proof of Corollary 2.

c) Lower and Upper Bound Algorithms:

Proposition 7: The global optimum of $\mathcal{L}(\widehat{\mathbf{Z}}, \mu)$ in (30) occurs at $\widehat{\mathbf{Z}}^{\bullet}=\operatorname{diag}\left(\left[z_{1}^{\bullet}, z_{2}^{\bullet}, \ldots, z_{n_{\mathrm{RN}}}^{\bullet}\right)\right.$, where

$$
z_{i}^{\bullet}=\frac{1}{2}\left[-\left(1+\frac{1}{v_{i}}\right)+\sqrt{\left(1-\frac{1}{v_{i}}\right)^{2}+\frac{4 W \gamma\left(v_{i}-1\right)}{\ln (2) v_{i} u_{i}^{\bullet}}}\right]_{+},
$$

$\forall i \in\left\{1, \ldots, n_{\mathrm{RN}}\right\}$, and $v_{i}$ are the eigenvalues of $\Upsilon$. Given that $P_{\mathrm{RN}}(\widehat{\mathbf{Z}})$ is expressed differently in (28) and (32) for the lower and upper bounds of (25), respectively, it implies that the values of $u_{i}^{\bullet}$ in (33) are also different for each of these bounds. For the lower bound, $u_{i}^{\bullet}$ represents the elements of the diagonal matrix $\widehat{\Lambda}^{-1} \widehat{\ddot{\Upsilon}}^{\uparrow}$ in (28). Whereas for the upper bound, $u_{i}^{\bullet}$ represents the diagonal elements of $\widehat{\Lambda}^{-1} \overline{\ddot{\Upsilon}}$ in (32). See section $\mathrm{G}$ of the Appendix for the proof. In addition, by inserting $\widehat{\mathbf{Z}} \bullet$ into $E_{b}(\widehat{\mathbf{Z}})$ in (30), the latter simplifies as

$$
E_{b}^{\bullet}=\frac{P_{c}^{\prime}+\sum_{i=1}^{n_{\mathrm{RN}}} z_{i}^{\bullet} u_{i}^{\bullet}}{R_{0}+W \sum_{i=1}^{n_{\mathrm{RN}}} \log _{2}\left(1+z_{i}^{\bullet} v_{i}\right)-\log _{2}\left(1+z_{i}^{\bullet}\right)},
$$

where $E_{b}^{\bullet}=E_{b}\left(\widehat{\mathbf{Z}}=\widehat{\mathbf{Z}}^{\bullet}\right)$.

The value of $\gamma$ in (33) is dependent on the value of $P_{\mathrm{RN}}\left(\widehat{\mathbf{Z}}^{\bullet}\right)$. If $P_{\mathrm{RN}}\left(\widehat{\mathbf{Z}}^{\bullet}\right)<P_{\mathrm{RN}}^{\max }$, i.e. unconstrained case, then $\gamma=E_{b}^{\bullet}$ in (33) (see Section $G$ of the Appendix for mode details). In this case, the problem of finding $\widehat{\mathbf{Z}} \bullet$ boils down to finding the variable $E_{b}^{\bullet}$ that satisfies equation (34). This is a univariate root-finding problem, since (34) is expressed solely as a function $E_{b}^{\bullet}$ (via $z_{i}^{\bullet}$ ), that can be solved in a lowcomplexity manner by using a classic univariate root-finding algorithm. On the contrary, if $P_{\mathrm{RN}}\left(\widehat{\mathbf{Z}}^{\bullet}\right) \geq P_{\mathrm{RN}}^{\max }$, i.e. power constrained case, then $\gamma=\mu^{\bullet}$ in (33) (see Section G of the Appendix for mode details), with $\mu^{\bullet}$ being a variable to optimise. Thus, the problem of finding $\widehat{\mathbf{Z}}^{\bullet}$ becomes equivalent to finding the variables $\mu^{\bullet}$ that satisfies the following power constraint equation (obtained by inserting $\widehat{\mathbf{Z}}^{\bullet}$ into (28) or (32))

$$
\frac{1}{\Delta_{\mathrm{RN}}} \sum_{i=1}^{n_{\mathrm{RN}}} z_{i}^{\bullet} u_{i}^{\bullet}=P_{\mathrm{RN}}^{\max },
$$

i.e. a univariate root-finding problem since (35) is expressed solely as a function $\mu^{\bullet}\left(\right.$ via $z_{i}^{\bullet}$ ) in this case. Hence, $\mu^{\bullet}$ can be computed in a low-complexity manner by using a root-finding algorithm (e.g. Newton-Raphson method [34]).

Given that the lower and upper bounds of the original Lagrangian can be both formulated as in (30), it implies that $E_{b}^{-}$and $E_{b}^{+}$can be computed by using a similar procedure, which is summarized in Algorithm 2; $E_{b}^{-}=E_{b}^{\bullet}$ if $u_{i}^{\bullet}$ are the elements of $\widehat{\boldsymbol{\Lambda}}^{-1} \widehat{\hat{\boldsymbol{\Upsilon}}}^{\uparrow}$ in (28), or $E_{b}^{+}=E_{b}^{\bullet}$ if $u_{i}^{\bullet}$ are the diagonal elements of $\widehat{\Lambda}^{-1} \overline{\ddot{\Upsilon}}$ in (32). 


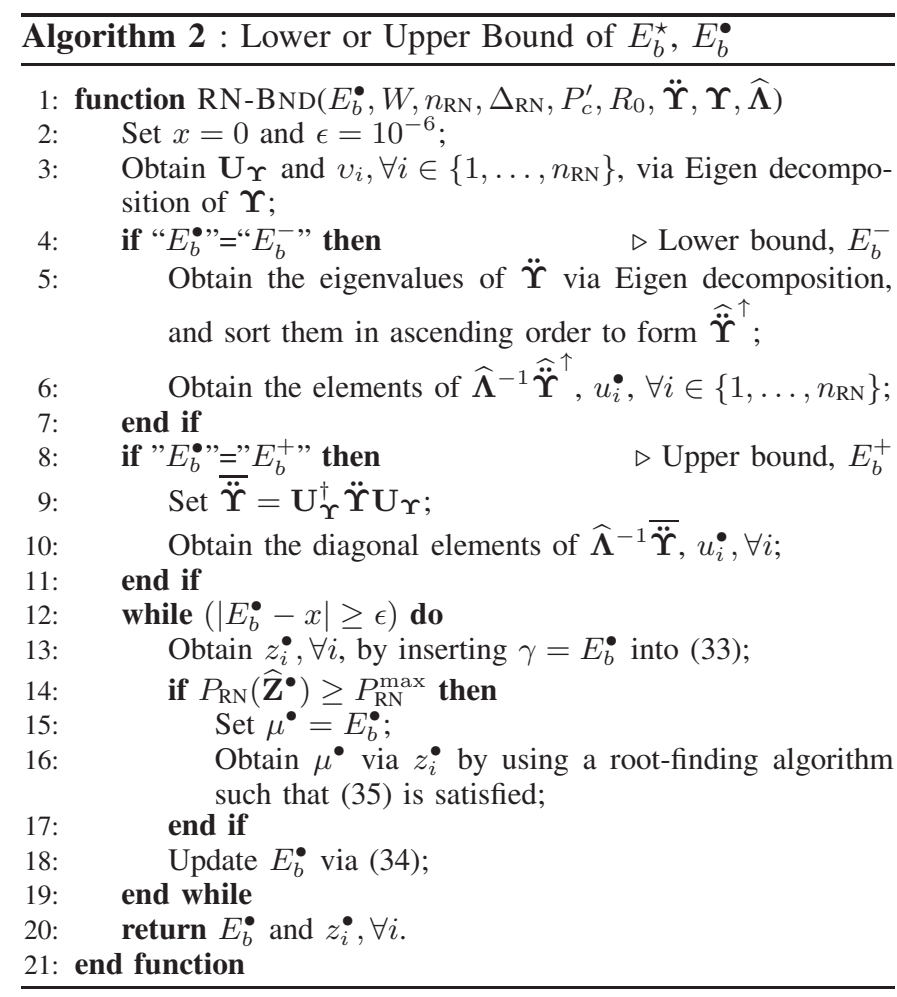

\section{2) EE-Optimal Relay Precoder:}

Proposition 8: Let $\varepsilon$ be a nonnegative real number that increases in an infinitesimal manner from 0 to infinity; then, the first value of $\varepsilon, \varepsilon^{\star}$, for which the equality

$$
E_{b}^{-}+\varepsilon=E_{b}\left(\mathbf{G}=\mathbf{G}\left(E_{b}^{-}+\varepsilon\right)\right),
$$

when $P_{\mathrm{RN}}\left(\mathbf{G}=\mathbf{G}\left(E_{b}^{-}+\varepsilon\right)\right) \leq P_{\mathrm{RN}}^{\max }$, is satisfied is the global minimum of $E_{b}$, such that $E_{b}^{\star}=E_{b}^{-}+\varepsilon^{\star}$. The EE-optimal relay precoding matrix, $\mathbf{G}^{\star}$, is then such that $\mathbf{G}^{\star}=\mathbf{G}\left(E_{b}^{-}+\right.$ $\left.\varepsilon^{\star}\right)$, where the latter is a solution of $\nabla_{\mathbf{G}} E_{b}\left(\mathbf{G}=\mathbf{G}^{\star}\right)=$

$$
\frac{1}{R_{\Sigma}\left(\mathbf{G}^{\star}\right)}\left[\nabla_{\mathbf{G}} P_{\Sigma}\left(\mathbf{G}=\mathbf{G}^{\star}\right)-E_{b}^{\star} \nabla_{\mathbf{G}} R_{\Sigma}\left(\mathbf{G}=\mathbf{G}^{\star}\right)\right]=\mathbf{0}
$$

with $P_{\mathrm{RN}}\left(\mathbf{G}=\mathbf{G}^{\star}\right) \leq P_{\mathrm{RN}}^{\max }$. In addition, $\nabla_{\mathrm{G}} P_{\Sigma}(\mathbf{G})$ and $\nabla_{\mathbf{G}} R_{\Sigma}(\mathbf{G})$ are expressed in (70). See section $\mathrm{H}$ of the Appendix for the proof.

Based on this proposition, the EE-optimal relay precoding matrix, $\mathbf{G}^{\star}$, can be obtained via Algorithm 3 in conjunction with, for instance, a gradient search (in the unconstrained case) or projected gradient search (in the power constrained case) method for obtaining $\mathbf{G}\left(E_{b}^{-}+\varepsilon^{\star}\right)$ for a given value of $\varepsilon^{\star}$. The search for the optimal $\varepsilon^{\star}$ stops when the difference between $E_{b}^{\star}$ and $E_{b}^{-}+\varepsilon^{\star}$ becomes negligible (i.e. when both equations (36) and (37) are jointly satisfied). Note that $w$ in line 5 of Algorithm 3 is an accuracy parameter, which is utilized to implement in practice the "infinitesimal" increasing of $\varepsilon$ from 0 to $\varepsilon^{\star}$; the larger $w$ is, the more accurate is the algorithm, but then the more iterations are likely to be required (i.e. increased complexity).

3) Low-complexity Energy-Efficient Relay Precoder:

Proposition 9: Contrary to $\mathbf{G}^{\star}$, which can only be obtained via a numerical method, the solution of the optimization
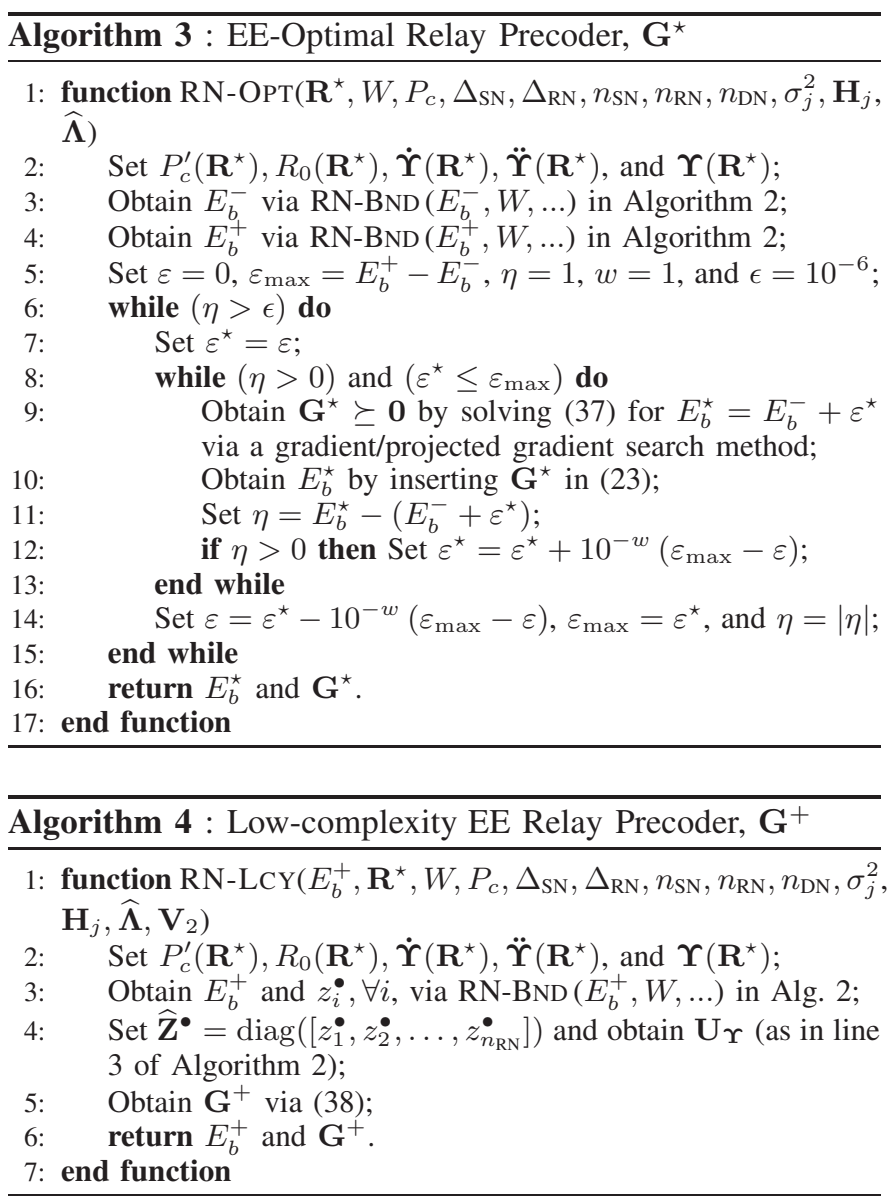

problem in (31), $\mathbf{G}^{+}$, can be expressed in closed-form as

$$
\mathbf{G}^{+}=\mathbf{V}_{2}\left(\frac{\sigma_{2}^{2}}{\sigma_{1}^{2}} \widehat{\mathbf{Z}}^{\bullet} \widehat{\boldsymbol{\Lambda}}^{-1}\right)^{\frac{1}{2}} \mathbf{U}_{\Upsilon}^{\dagger},
$$

where $\widehat{\mathbf{Z}}^{\bullet}=\operatorname{diag}\left(\left[z_{1}^{\bullet}, z_{2}^{\bullet}, \ldots, z_{n_{\mathrm{RN}}}\right]\right)$, and $z_{i}^{\bullet}$ is defined as in (33) with $u_{i}^{\bullet}$ being the diagonal elements of $\widehat{\Lambda}^{-1} \overline{\ddot{\Upsilon}}$.

Proof: Given that $\widehat{\mathbf{Z}}=\frac{\sigma_{1}^{2}}{\sigma_{2}^{2}} \widehat{\mathbf{G}} \widehat{\mathbf{\Lambda}}$ and $\mathbf{G}^{+}$minimizes (31), i.e. (23) subject to $\mathbf{G}=\mathbf{V}_{2} \widehat{\mathbf{G}}^{\frac{1}{2}} \mathbf{U}_{\Upsilon}^{\dagger}$, it implies that $\mathbf{G}^{+}$can be defined as in (38).

Accordingly, $\mathbf{G}^{+}$, can be obtained via Algorithm 4, which has a lower computational complexity than Algorithm 3. Indeed, it only involves one or two univariate root-finding search(es) since it is based on Algorithm 2. However, it is not necessary optimal in general, given that the search domain is restricted to the domain of positive semi-define matrix having a particular structure.

\section{Joint Source and Relay Precoding Optimization}

1) Alternating Optimization Procedure: As in [10], [16], [20], [24], the main optimization problem in (9) can be solved by using an alternating optimization procedure based on $\mathbf{R}^{\star}$ and $\mathbf{G}^{\star}$ or $\mathbf{G}^{+}$, as follows:

1) Obtain $\widehat{\Lambda}$ and $V_{2}$ via singular value decomposition. Then, set $\mathbf{R}^{\star^{(1)}}=\mathbf{I}_{n_{\mathrm{SN}}}, \mathbf{G}^{\star^{(1)}}$, and obtain the eigenvalues of $\boldsymbol{\Psi}\left(\Delta_{\mathrm{SN}}, \Delta_{\mathrm{RN}}, \mathbf{G}^{\star^{(1)}}\right), \psi_{i}\left(\Delta_{\mathrm{SN}}, \Delta_{\mathrm{RN}}\right)$. As in [10], we consider $N$ different randomly selected initialization matrices $\mathbf{G}^{\star^{(1)}} \succeq \mathbf{0}$. Next, set $E_{b}^{\star}=1+\frac{\ln (2)}{W} \max _{i}\left\{\psi_{i}\right\}$. 
2) At the $k$-th iteration, $\mathbf{G}^{\star^{(k)}}$ is used as an input of Algorithm 1, which updates the value of $E_{b}^{\star}$ and return $\mathbf{R}^{\star^{(k+1)}}$.

3) Next, $\mathbf{R}^{\star^{(k+1)}}$ is used as an input of Algorithm 3, which updates the value of $E_{b}^{\star}$ and return $\mathbf{G}^{\star^{(k+1)}}$.

4) Steps 2) and 3) are repeated iteratively until convergence, i.e. until the values of $E_{b}^{\star}$ at the end of the $k$-th and $k+1$-th iterations are the same.

Note that the same procedure can also be used for obtaining $\mathbf{G}^{+}$instead of $\mathbf{G}^{\star}$, where Algorithm 4, instead of Algorithm 3 , is used at step 3 ).

2) Convergence Discussion and Results: Following the same line of reasoning as in [24], the convergence of the alternating procedure for solving (9) in the previous subsection can be established as follows; first, given that the optimization problem in (9) has a unique global minimum when the variable $\mathbf{R}$ is optimized for a known $\mathbf{G}$, it implies that $E_{b}\left(\mathbf{R}^{\star^{(k+1)}}, \mathbf{G}^{\star^{(k)}}\right) \leq E_{b}\left(\mathbf{R}^{\star^{(k)}}, \mathbf{G}^{\star^{(k)}}\right)$, at the $k$-th iteration. Second, since the optimization problem in (9) has a unique global minimum when the variable $\mathbf{G}$ is optimized for a known $\mathbf{R}$, it implies that $E_{b}\left(\mathbf{R}^{\star^{(k+1)}}, \mathbf{G}^{\star^{(k+1)}}\right) \leq E_{b}\left(\mathbf{R}^{\star^{(k+1)}}, \mathbf{G}^{\star^{(k)}}\right)$, at the $k$-th iteration. By combining the two previous inequalities, such that $E_{b}\left(\mathbf{R}^{\star^{(k+1)}}, \mathbf{G}^{\star^{(k+1)}}\right) \leq E_{b}\left(\mathbf{R}^{\star^{(k)}}, \mathbf{G}^{\star^{(k)}}\right)$, and knowing that $E_{b}$ is lower bounded, we can conclude that the conditional updating of $\mathbf{R}^{\star}$ (for a fixed $\mathbf{G}^{\star}$ ) and $\mathbf{G}^{\star}$ (for a fixed $\mathbf{R}^{\star}$ ) at each iteration either decreases or maintains the value of $E_{b}^{\star}=E_{b}\left(\mathbf{R}^{\star}, \mathbf{G}^{\star}\right)$. In other words, $E_{b}^{\star}$ is the best local minimum of $E_{b}$, for a given $\mathbf{R}^{\star}$ or $\mathbf{G}^{\star}$, at each iteration.

However, similar to [10], [16], [20], it cannot be guaranteed that $E_{b}^{\star}$ is always the global minimum of (9) in the general case since $E_{b}(\mathbf{R}, \mathbf{G})$ in (9) is not necessarily jointly strictly pseudo-convex/convex in both $\mathbf{R}$ and $\mathbf{G}$. Nevertheless, in the following special cases, $E_{b}^{\star}$ is guaranteed to be the global minimum of (9):

- if $\sigma_{0}^{2} \ll 1, \sigma_{1}^{2} \gg 1$, or $\sigma_{2}^{2} \gg 1$, then (9) becomes equivalent to a DL only EE-based optimization problem (i.e. independent of $\mathbf{G})$, such that $E_{b}\left(\mathbf{R}^{\star}, \mathbf{G}^{\star}=\mathbf{0}\right)$ is optimal;

- if $\sigma_{2}^{2} \ll 1$, then (9) becomes independent of $\mathbf{G}$ as in the previous case;

- if $\sigma_{2}^{2} \ll 1$ and $\sigma_{1}^{2} \ll 1$, then $\mathbf{R}$ and $\mathbf{G}$ become uncorrelated in (1). In turn, it has been discussed in [10] (for the RL only scenario, i.e. $\sigma_{0}^{2} \gg 1$ ) that it is a sufficient condition for (9) to converge to a global optimum.

Figure 2 depicts the number of iterations that are necessary for the alternating optimization procedure to convergence in the three special cases previously discussed, i.e. $\sigma_{0}^{2}=-30$ $\mathrm{dB}$ and $\sigma_{1}^{2}=\sigma_{2}^{2}=0 \mathrm{~dB}\left(\Leftrightarrow \sigma_{0}^{2} \ll 1\right), \sigma_{2}^{2}=-30 \mathrm{~dB}$ and $\sigma_{0}^{2}=\sigma_{1}^{2}=0 \mathrm{~dB}\left(\Leftrightarrow \sigma_{2}^{2} \ll 1\right)$, or $\sigma_{1}^{2}=\sigma_{2}^{2}=-30 \mathrm{~dB}$ and $\sigma_{0}^{2}=0 \mathrm{~dB}\left(\Leftrightarrow \sigma_{2}^{2} \ll 1\right.$ and $\left.\sigma_{1}^{2} \ll 1\right)$, respectively. In addition, a case where convergence is not necessarily guaranteed, i.e. $\sigma_{0}^{2}=20 \mathrm{~dB}$ and $\sigma_{1}^{2}=\sigma_{2}^{2}=10 \mathrm{~dB}$, is also plotted. Note that the alternating procedure is run $N=10$ times (such that each subplot has 10 dashed lines), where the random initialization matrix $\mathbf{G}^{\star^{(1)}}$ is different for each run. The results clearly show that the alternating procedure converges towards

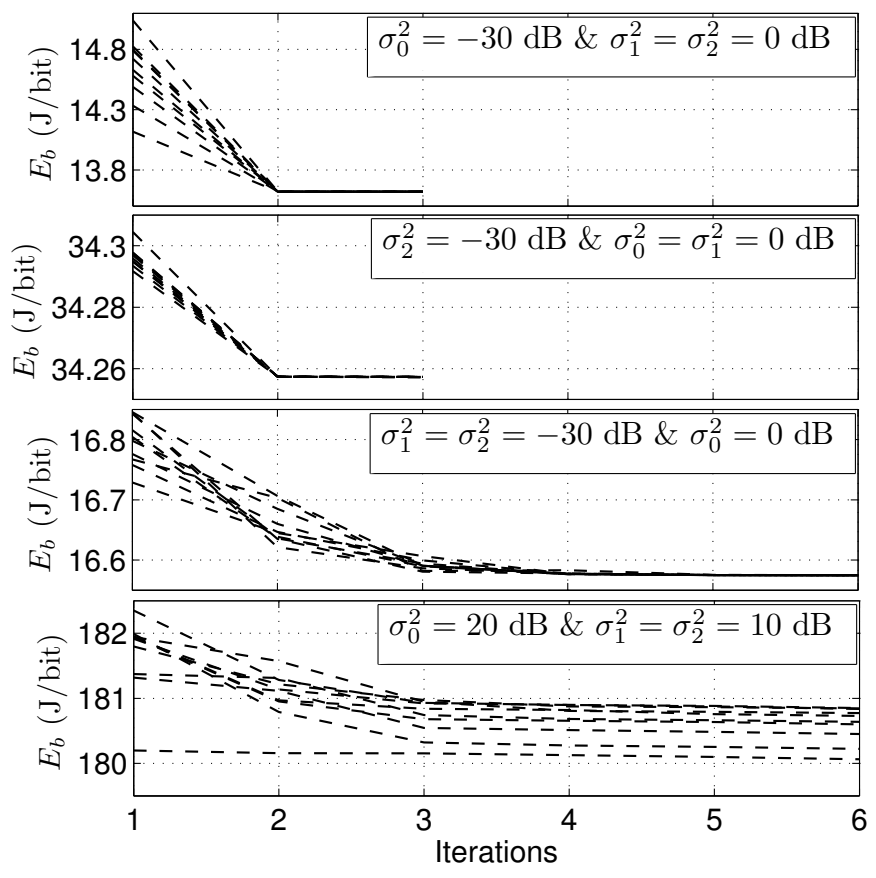

Fig. 2: Number of iterations required for the alternating optimization procedure to converge in different settings.

the same value and, hence, is independent of $\mathbf{G}^{\star^{(1)}}$, when considering any of the three special case settings. In turn, this confirms that the alternating procedure can reach the global minimum in these special cases, regardless of $\mathbf{G}^{\star^{(1)}}$. Whereas in the case of $\sigma_{0}^{2}=20 \mathrm{~dB}$ and $\sigma_{1}^{2}=\sigma_{2}^{2}=10 \mathrm{~dB}$, the outcome of the alternating procedure is clearly dependent of $\mathrm{G}^{\star^{(1)}}$, i.e. different $\mathbf{G}^{\star^{(1)}}$ provide different outcomes. Thus, this justifies the use of multiple initialization points [10] for increasing the likelihood of reaching the global minimum of (9).

\section{Numerical RESUlts AND Discussions}

Our simulations, which are averaged over 10000 runs, assume a downlink transmission of the cooperative MIMO AF system, such that the SN is a BS and the DN is a UE. They also rely on the power model parameters of Table 1 of [12] (but with $P_{\mathrm{SN}}^{\mathrm{SlpA}}=0 \mathrm{~W}$ ) for setting values to all the parameters discussed in Section II-B. In addition, note that $P^{\max }$ per antenna is between 6 to $20 \mathrm{~W}$ for a typical micro/macro BS [4], and between 1 to $5 \mathrm{~W}$ for a typical urban/rural RN [26]. We also consider a single-tap i.i.d MIMO Rayleigh fading channel between each node, a unit bandwidth $(W=1)$, and $\varsigma=1 / 2$ in $P_{c}$. Moreover, we assume that all the channel matrices have the same dimension, i.e. $n=n_{\mathrm{SN}}=n_{\mathrm{RN}}=n_{\mathrm{DN}}$, and $N=10$ in step 1) of Section III-C1.

\section{A. Performance Results and Insights}

In order to demonstrate the benefits, in terms of EE, of our source and relay precoding optimization scheme for cooperative MIMO-AF systems, we compare its performance against existing approaches that either minimize the MSE [20], maximize the SE [16], or minimize $E_{b}$ [23] in the same scenario (when considering both direct and relay links), and 

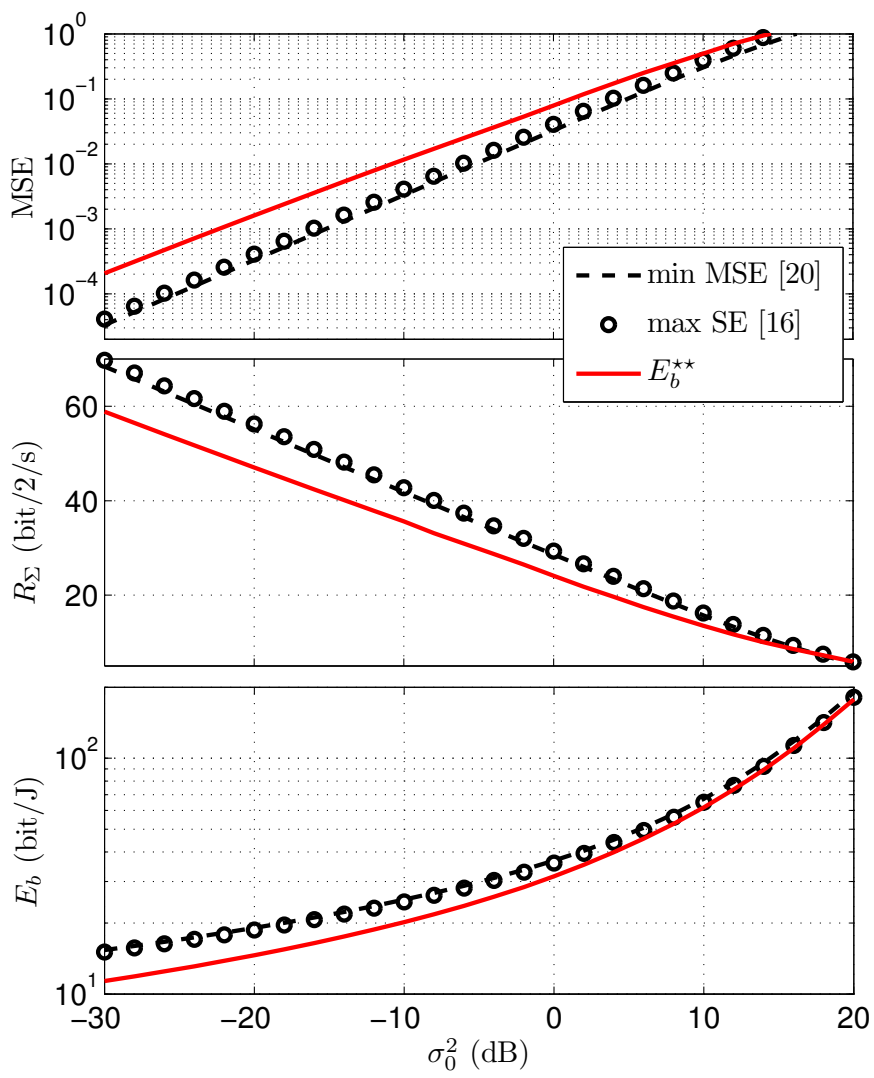

Fig. 3: MSE, sum-rate, and EE performances of our scheme, against other existing non-EE-based cooperative MIMO-AF precoding schemes, as a function of $\sigma_{0}^{2} \mathrm{~dB}$.

other existing $\mathrm{EE} / E_{b}$-based approaches that either optimize the EE of the DL [35] or RL [10], [11] on its own . For ease of introduction, the different schemes that are compared in this section are denoted, as follows

- $E_{b}^{\star \star}=E_{b}\left(\mathbf{R}^{\star}, \mathbf{G}^{\star}\right)$ is the outcome of our EE-optimal source and relay precoding design, which can be implemented through Algorithms 1 and 3.

- $E_{b}^{\star+}=E_{b}\left(\mathbf{R}^{\star}, \mathbf{G}^{+}\right)$is the outcome of our EE-optimal source and low-complexity relay precoding design, which can be implemented through Algorithms 1 and 4.

- min MSE is the outcome of the MSE-optimal source and relay precoding design proposed in [20].

- $\max \mathrm{SE}$ is the outcome of the SE-based source and relay precoding design proposed in [16].

- $E_{b}^{\star \diamond}$ denotes the outcome of our preliminary work in [23], where the EE-optimal source precoding is utilized in conjunction with a different relay precoding structure, i.e. $\mathbf{G}=\mathbf{V}_{2} \widehat{\mathbf{G}}^{\frac{1}{2}} \mathbf{U}_{1}^{\dagger}$.

- $E_{b}^{\star \circ}=\min _{\mathbf{R}} E_{b}(\mathbf{R}, \mathbf{G}=\mathbf{0})$ is the outcome of the EEoptimal source precoding design for DL only in [35].

- $E_{b}^{* *}=\min _{\mathbf{R}, \mathbf{G}} \lim _{\sigma_{0} \rightarrow \infty} E_{b}(\mathbf{R}, \mathbf{G})$ is the outcome of the EE-optimal source and relay precoding design for RL only in [10], [11].

Note that when comparing the EE of cooperative schemes with DL or RL only scheme, it is necessary to integrate the fact that $P_{c}$, which accounts for all the fixed circuit consumed powers in the system, is different in each case. In the cooperative case,

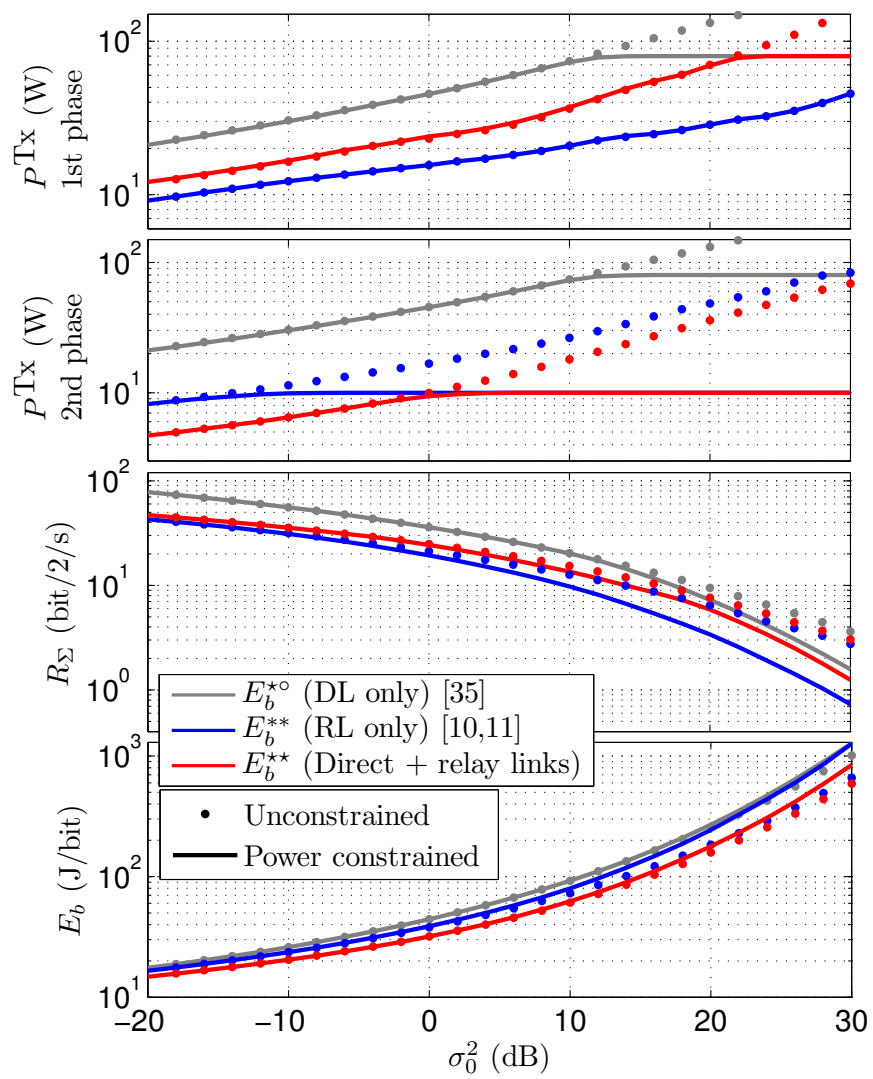

Fig. 4: Transmit power, sum-rate, and EE performances of our scheme against other existing EE-based DL only and RL only schemes, as a function of $\sigma_{0}^{2} \mathrm{~dB}$.

$P_{c}$ is defined right after equation (6), whereas in the DL or RL only case, $P_{c}=n_{\mathrm{SN}} P_{\mathrm{SN}}^{\mathrm{CipA}}+P_{\mathrm{SN}}^{\mathrm{Ci}}+\varsigma\left(n_{\mathrm{DN}} P_{\mathrm{DN}}^{\mathrm{CipA}}+P_{\mathrm{DN}}^{\mathrm{Ci}}\right)$ or $P_{c}=n_{\mathrm{SN}}\left(P_{\mathrm{SN}}^{\mathrm{CipA}}+P_{\mathrm{SN}}^{\mathrm{SlpA}}\right)+P_{\mathrm{SN}}^{\mathrm{Ci}}+(1+\varsigma)\left(n_{\mathrm{RN}} P_{\mathrm{RN}}^{\mathrm{CipA}}+P_{\mathrm{RN}}^{\mathrm{Ci}}\right)+$ $\varsigma\left(n_{\mathrm{DN}} P_{\mathrm{DN}}^{\mathrm{CipA}}+P_{\mathrm{DN}}^{\mathrm{Ci}}\right)+n_{\mathrm{DN}} P_{\mathrm{DN}}^{\mathrm{SlpA}}$, respectively.

Figure 3 compares the MSE, sum-rate, and EE performances of our scheme, against other existing precoding schemes that are dedicated to either minimize the MSE [20] or maximize the SE [16] of cooperative MIMO-AF systems, for $n=4$, $P_{\mathrm{SN}}^{\max }=80 \mathrm{~W}, P_{\mathrm{RN}}^{\max }=10 \mathrm{~W}, \sigma_{1}^{2}=\sigma_{2}^{2}=\sigma_{0}^{2} / 10$. Note that the unit for sum-rate is $b i t / 2 / s$, since we consider the number of transmitted bits over two transmission phases. As it is expected, the precoding schemes of [20] and [16] provide the best MSE and sum-rate performances, respectively, whereas our scheme provides the best energy consumption. Indeed, our scheme can reduce $E_{b}$ by up to $25 \%$ in good channel condition (when $\sigma_{0}^{2}=-30 \mathrm{~dB}$ ), but at the expense of a $15 \%$ reduction in sum-rate when compared to max $\mathrm{SE}$ and almost one order of MSE magnitude when compared to min MSE. It can also be remarked that as the channel condition worsen (when $\sigma_{0}^{2}$ increases), as the results of the three schemes start to converge. In turn, this indicates that the type of precoding design becomes less of an issue in poor channel condition.

Figure 4 compares the transmit power (in both transmission phases), sum-rate, and EE performances of our scheme, against other existing $\mathrm{EE} / E_{b}$-based approaches that either optimize the EE of the DL [35] or RL [10], [11] on its own, for $n=4$, $P_{\mathrm{SN}}^{\max }=80 \mathrm{~W}, P_{\mathrm{RN}}^{\max }=10 \mathrm{~W}, \sigma_{1}^{2}=\sigma_{2}^{2}=\sigma_{0}^{2} / 10$. Note 
that both the unconstrained and power constrained results are plotted for each scheme. In terms of $E_{b}$ performance, the results show that our new scheme can outperform the two other schemes by more than $30 \%$ in both the unconstrained and power constrained scenarios. Whereas in terms of sum-rate, it can be remarked that in this particular setting, the DL only transmission scheme [35] performs better than our cooperative scheme or the RL only scheme, by up to $40 \%$. This is similar to the results in Fig. 3 and it indicates the existence of a tradeoff between EE and rate [30]. Even though DL transmission provides a better rate than cooperative transmission, this does not translates in a lower energy consumption. Indeed, the transmit power results indicate that the DL transmission always utilizes more transmit power than the two other schemes (up to 8 times more in the 2nd transmission phase) and, in turn, this explains why it is less energy efficient than our cooperative scheme. By analyzing the four subplots together, we can conclude that our scheme provides the best trade-off between power and rate, such that it exhibits the best $E_{b}$.

The transmit power results of Fig. 4 are also very informative about the way in which EE-based optimization works and differs from SE maximization or MSE minimization, which echoes the first paragraph of Section III. Indeed, the top two subplots of Fig. 4 show that depending on the value of $\sigma_{0}^{2}$, the optimization problem in (9) is either unconstrained (for $\sigma_{0}^{2} \leq 2$ $\mathrm{dB}$ ), SPC at the RN (for $2 \mathrm{~dB}<\sigma_{0}^{2} \leq 22 \mathrm{~dB}$ ) or DPC (for $\sigma_{0}^{2}>$ $22 \mathrm{~dB}$ ). Moreover, as it was expected, $E_{b}^{\star \star}$ is always lower in the unconstrained rather than in the constrained regime. In contrast, the minimum MSE and maximum SE are always achieved when both power constraints are met (DPC regime) in Fig. 3. This explains why $E_{b}^{\star \star}$ tends to converge towards min MSE and max EE in Fig. 3 when $\sigma_{0}^{2} \gg 0$, since in this case, all the schemes operate in the DPC regime.

Figure 5 depicts the EE gain, $\Delta_{\mathrm{EE}}$, of our novel EEbased cooperative precoding scheme against EE-based noncooperative precoding schemes for various $\sigma_{i}^{2}$ values, $\forall i \in$ $\{0,1,2\}, n=4, P_{\mathrm{SN}}^{\max }=80 \mathrm{~W}, P_{\mathrm{RN}}^{\max }=10 \mathrm{~W}$, and where

$$
\Delta_{\mathrm{EE}}=100\left[1-E_{b}^{\star \star} / \chi\right]_{+} \% \text {. }
$$

Note that $\chi=\min \left\{E_{b}^{\star \circ}, E_{b}^{* *}\right\}$ in Fig. 5. The results clearly confirm the benefits, in terms of EE, of joint source and relay precoding optimization in comparison with existing approaches, where precoding matrices are optimized for either the direct or relay link separately. Indeed, $45 \%$ or more of each subplot area has a $\Delta_{\mathrm{EE}} \geq 0$, which graphically indicates that joint source and relay precoding matrices optimization is useful for improving the EE of MIMO-AF systems. For instance, in Fig. 5 b) and c), an EE improvement of up to $28 \%$ is achieved. However, it can be remarked that the intensity of the gain is not uniform; it clearly depends on the values of $\sigma_{i}^{2}$, which themselves reflect the quality of each links. Similar observations have also been reported in [17], [18], for the case of SE-based joint source and relay precoding subject to power constraints. On the one hand, we know that multi-hop communication (RL only) is prone to the 'bottleneck' effect (see Fig. 4 of [15]), where the overall rate of the RL is limited by the rate of its worst hop (see Section IV. B1) of [11]). Hence, whenever the DL exhibits a far better link quality than

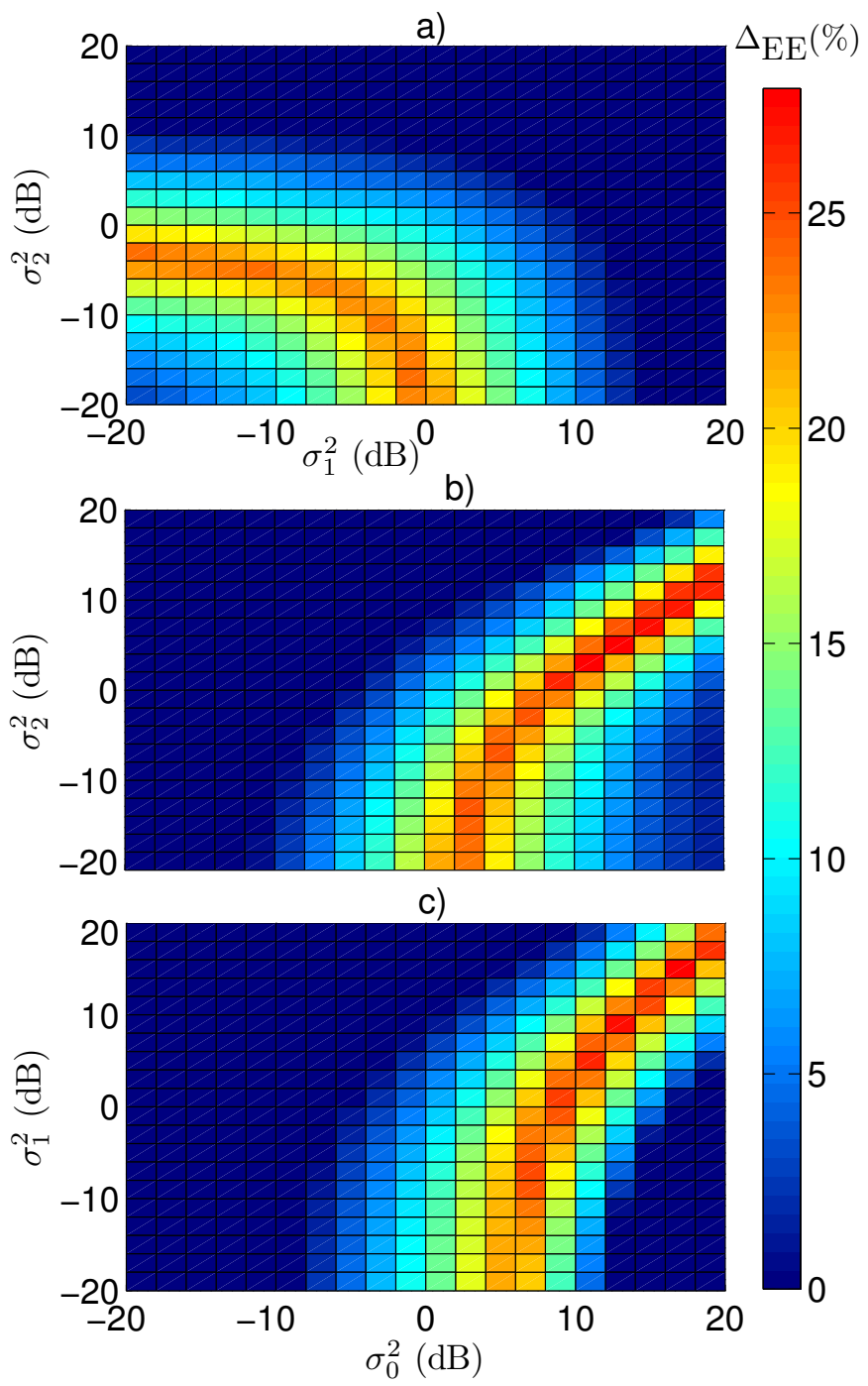

Fig. 5: EE gain comparison of our scheme against other existing EE-based DL only and RL only schemes, as a function of a) $\left(\sigma_{1}^{2}, \sigma_{2}^{2}\right)$ when $\left.\sigma_{0}^{2}=0 \mathrm{~dB}, \mathrm{~b}\right)\left(\sigma_{0}^{2}, \sigma_{2}^{2}\right)$ when $\sigma_{1}^{2}=0 \mathrm{~dB}$, and c) $\left(\sigma_{0}^{2}, \sigma_{1}^{2}\right)$ when $\sigma_{2}^{2}=0 \mathrm{~dB}$.

the worst link of the two-hop RL, i.e. $\sigma_{0}^{2}<<\max \left\{\sigma_{1}^{2}, \sigma_{1}^{2}\right\}$, the precoding at the $\mathrm{SN}$ prioritizes the DL transmission such that $E_{b}^{\star \star} \approx E_{b}^{\star \circ}$. In turn, this explains why $\Delta_{\mathrm{EE}} \simeq 0$ in the left region of both Fig. 5 b) and c), as well as in the upper right region of Fig. 5 a). On the other hand, whenever the RL exhibits a far better link quality than the DL, i.e. $\sigma_{0}^{2}>\max \left\{\sigma_{1}^{2}, \sigma_{1}^{2}\right\}$, the precoding at both the SN and RN prioritize the RL transmission such that $E_{b}^{\star \star} \approx E_{b}^{* *}$. As a result, $\Delta_{\mathrm{EE}} \simeq 0$ in the lower right region of both Figs. 5 b) and c), as well as in the lower left region of Fig. 5 a). Note that similar results have been remarked for higher number of antennas, e.g. $n=8$ or $n=16$. Finally, the link quality being dependent on the RN placement in a practical deployment, Fig. 5 is quite informative for getting the most out of cooperative MIMO-AF communication systems in terms of EE.

Figures 6 and 7 complement the results of Fig. 5 by focusing on the impact of power constraints and imperfect CSI, respectively, on the EE gain. By considering the same 


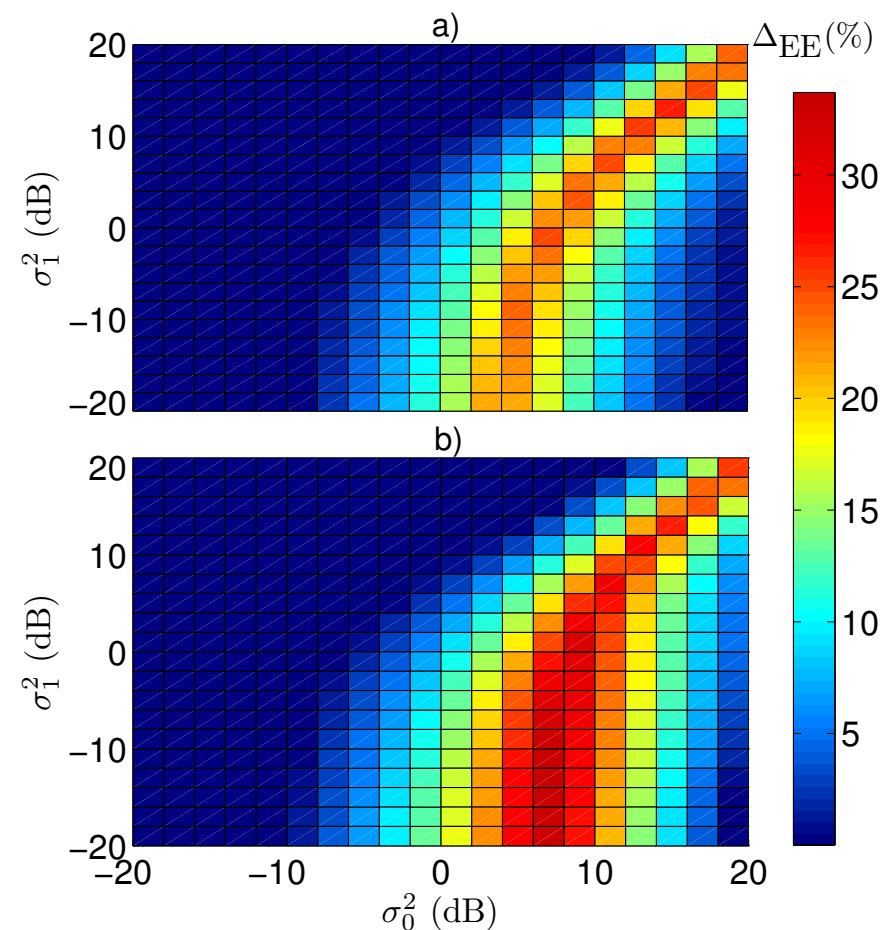

Fig. 6: EE gain comparison of our scheme against other existing EE-based DL only and RL only schemes, for different power constraints.

settings as in Fig. 5 c), except that $P_{\mathrm{SN}}^{\max }=40 \mathrm{~W}, P_{\mathrm{RN}}^{\max }=20$ $\mathrm{W}$ in Fig. 6 a) and $P_{\mathrm{SN}}^{\max }=20 \mathrm{~W}, P_{\mathrm{RN}}^{\max }=5 \mathrm{~W}$ in Fig. $6 \mathrm{~b}$ ), Fig. 6 further confirms that our scheme can be more energy efficient than existing approaches and that the improvement is localized. In addition, it can be remarked that more EE gain, around $33 \%$, is achieved in Fig. 6 b) when the power constraints are more stringent, i.e. $P_{\mathrm{SN}}^{\max }=20 \mathrm{~W}, P_{\mathrm{RN}}^{\max }=5$ $\mathrm{W}$ instead of $P_{\mathrm{SN}}^{\max }=40 \mathrm{~W}, P_{\mathrm{RN}}^{\max }=20 \mathrm{~W}$ in Fig. 6 a) or $P_{\mathrm{SN}}^{\max }=80 \mathrm{~W}, P_{\mathrm{RN}}^{\max }=10 \mathrm{~W}$ in Fig. $5 \mathrm{c}$ ). Whereas in Fig. 7 , by relying on the noisy CSI model of [36] for modeling the imperfection in CSI estimation, the EE gain is depicted when considering the same settings as in Fig. $5 \mathrm{c}$ ), except that $\sigma_{E}^{2}=0.1$ in Fig. 7 a) and $\sigma_{E}^{2}=0.5$ in Fig. 7 b). In the noisy CSI framework of [36], $\sigma_{E}^{2}$ is used to model the quality of the CSI estimation; $\sigma_{E}^{2}=0$ being equivalent to the perfect CSI estimation case (e.g., as in Figs. 5 and 6), whereas the estimation quality degrades as $\sigma_{E}^{2}$ increases. Even though the absolute performance of all the schemes will obviously degrade as $\sigma_{E}^{2}$ increases, it is interesting to see in Fig. 7 that it is not the case for the relative performance of our novel EEbased cooperative precoding scheme against EE-based noncooperative precoding schemes. On the contrary, higher values of $\Delta_{\mathrm{EE}} \%$ can be achieved when $\sigma_{E}^{2}$ increases, i.e. an EE gain of up to $50 \%$ is obtained in Fig. 7 b) for $\sigma_{E}^{2}=0.5$. In turn, this indicates that our cooperative scheme is more resilient to CSI estimation error that the existing non-cooperative precoding schemes. This improved resilience could be due to diversity; the cooperative scheme relies on two different transmission routes, instead of one for the non-cooperative schemes. Finally, as in Figs. 5 and 6, the EE gain is still localized in Fig. 7.

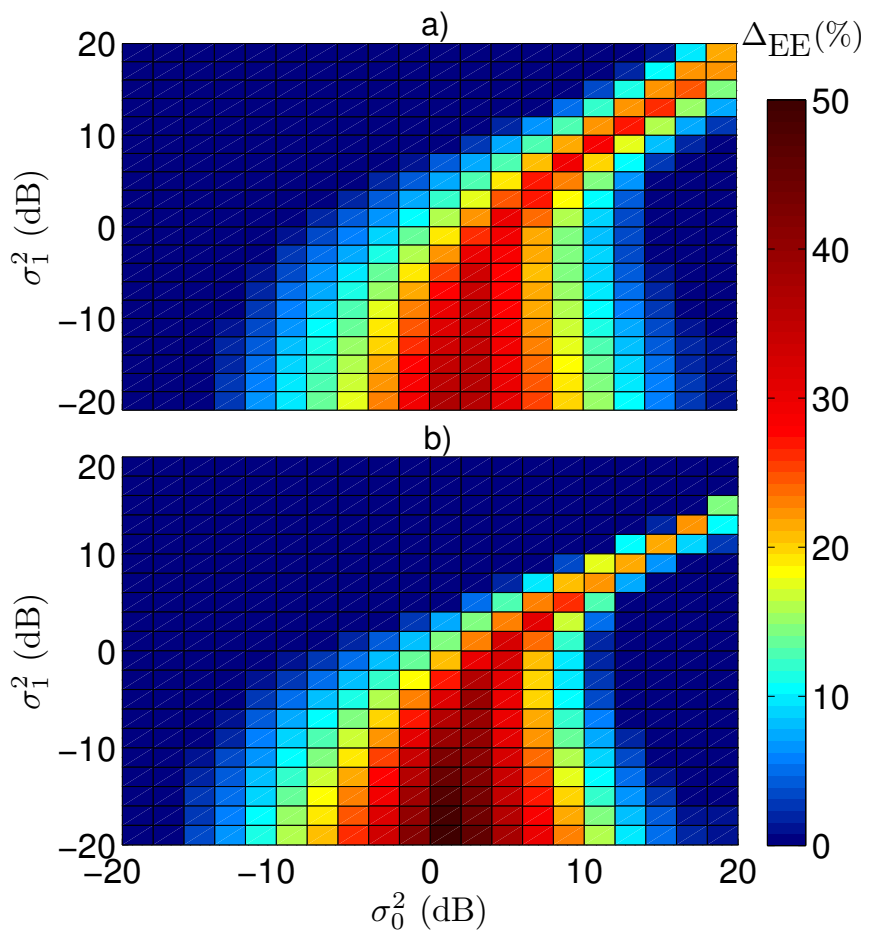

Fig. 7: EE gain comparison of our scheme against other existing EE-based DL only and RL only schemes, when considering imperfect CSI.

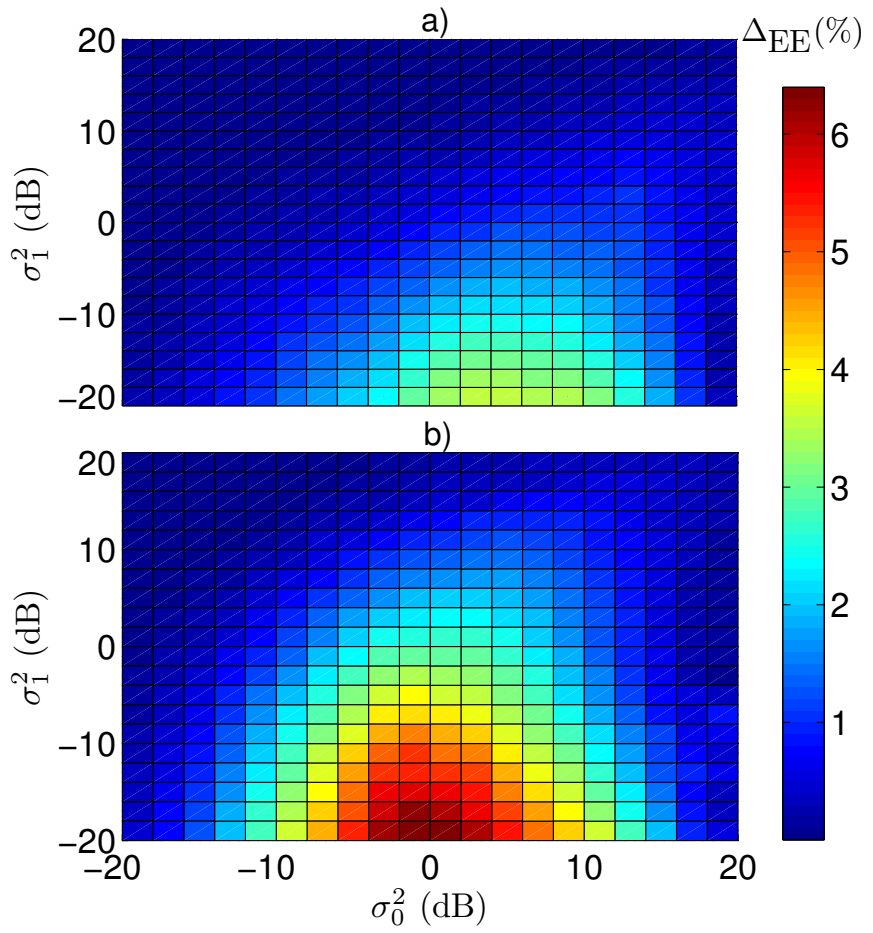

Fig. 8: EE gain comparison of our scheme against a) its low-complexity alternative and b) our preliminary EE-based precoding scheme of [23].

Figure 8 compares the EE performance of the our scheme with the EE-optimal relay precoder against the low-complexity energy efficient relay precoder in Fig. 8 a) and the relay 
precoder of [23] in Fig. 8 b), when considering the same settings as in Fig. 5 c). Consequently, $\Delta_{\mathrm{EE}}$ is defined as in (39), but where $\chi=E_{b}^{\star+}$ and $\chi=E_{b}^{\star \diamond}$ in Fig. 8 a) and b), respectively. On the one hand, Fig. 8 a) confirms that the relay precoding matrix $\mathbf{G}^{+}$is not as energy efficient as $\mathbf{G}^{\star}$ in the general case (since $\Delta_{\mathrm{EE}} \geq 0$ ); however, in this particular example, the difference of performance between using $\mathbf{G}^{\star}$ or $\mathbf{G}^{+}$remains below $4 \%$. On the other hand, Fig. 8 b) clearly indicates that the relay precoding structure $\mathbf{G}=\mathbf{V}_{2} \widehat{\mathbf{G}}^{\frac{1}{2}} \mathbf{U}_{1}^{\dagger}$, which is known to be optimal in various other MIMO-AF precoding scenarios [10], [11], [14], [25], is not optimal here since $E_{b}^{\star \diamond}$ can differ by more than $6 \%$ compared to $E_{b}^{\star \star}$.

\section{B. Complexity Analysis and Results}

In order to put the results in Figs. 5 and 8 into perspective, we discuss here the computational complexity of the different algorithms proposed in this paper, when assuming that $n=$ $n_{\mathrm{SN}}=n_{\mathrm{RN}}=n_{\mathrm{DN}}$ :

- Even though $\mathbf{R}^{\star}$ in Algorithm 1 is obtained through a combination of simple univariate and/or bivariate rootfinding searches (with complexity linear in $n$ ), the complexity of the whole algorithm is asymptotically driven by the complexity of the matrix operations it uses, e.g. eigen decomposition (ED) for obtaining $\mathbf{U}_{\Psi}$, matrix multiplication/inversion for computing $\dot{\Psi}, \ddot{\Psi}$, and $\mathbf{R}^{\star}$. Given that these matrix operations usually exhibit a computational complexity of $O\left(n^{3}\right)$, we expect Algorithm 1 to exhibit the same sort of asymptotical complexity.

- Algorithm 2 also utilizes univariate root-finding searches as well as ED and matrix multiplication/inversion; consequently, we also expect Algorithm 2 to exhibit a computational complexity of $O\left(n^{3}\right)$. However, Algorithm 2 uses at worst two root-finding searches (i.e. for unconstrained and DPC cases) instead of three in Algorithm 1. Thus, we expect Algorithm 2 to exhibit a lower computational complexity than Algorithm 1.

- Algorithm 4 uses Algorithm 2 to return $\mathbf{G}^{+}$, such that it follows the same structure as Algorithm 1. Hence, it is expected to have a similar complexity of $O\left(n^{3}\right)$.

- The EE-optimal relay precoding in Algorithm 3 is based on an iterative approach, where the average number of iterations, $N_{\text {iter }}$, that are necessary for finding $\mathbf{G}^{\star}$ are increasing with $w$ and $1 / \epsilon$; $w$ and $\epsilon$ being accuracy parameters that are defined at line 5 of Algorithm 3. The larger $w$ and $1 / \epsilon$ are, the more accurate is this algorithm, but the more iterations (complexity) are required. For instance, when $w=1$, then inner while loop in Algorithm 3 (lines 8 to 13 ) is perform at most 10 times for each outer while loop (lines 6 to 15). Meanwhile, inside the inner while loop, a gradient/projected gradient search is performed to update $\mathrm{G}^{\star}$, which again relies on matrix operations, such as determinant and multiplication, that exhibit a computational complexity of $O\left(n^{3}\right)$. Overall, the optimal approach is expected to exhibit a computational complexity of $O\left(N_{\text {iter }} n^{3}\right)$. In addition, given that Algorithm 3 also required to run Algorithm 2 twice, its computational complexity can only be always greater than the one of Algorithm 4.

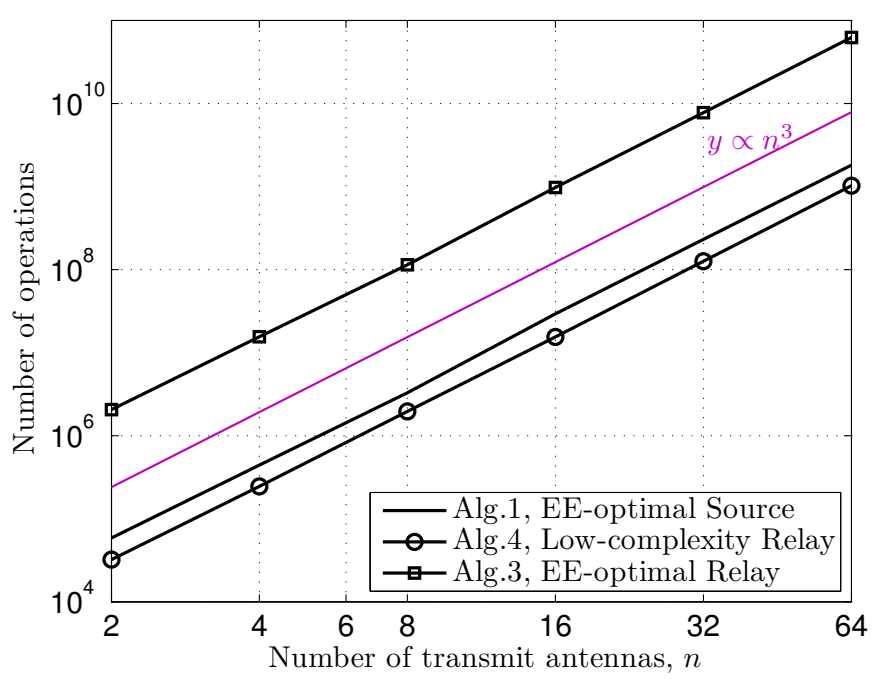

Fig. 9: Comparison of the average number of basic operations required by Algorithms 1,3 , and 4 as a function of $n$, for $\sigma_{0}^{2}=0 \mathrm{~dB}$ and $\sigma_{1}^{2}=\sigma_{2}^{2}=-6 \mathrm{~dB}$.

Figure 9 depicts the computational complexity, measured in terms of the average number of basic operations (e.g. addition, substraction, multiplications, etc.), of Algorithms 1, 3, and 4, as a function of the matrix dimension, $n$, for $\sigma_{0}^{2}=0 \mathrm{~dB}$ and $\sigma_{1}^{2}=\sigma_{2}^{2}=-6 \mathrm{~dB}$ (i.e. a setting for which the $\mathrm{EE}$ gain is significant according to Fig. 5 a)). The results first confirm that the complexity of all three algorithms is asymptotically driven by the complexity of the matrix operations, i.e. $n^{3}$, and, as it was expected, that Algorithm 4 is the least complex of the three algorithms; Algorithm 4 is roughly 60 times less complex than Algorithm 3. Hence, the low-complexity approach of Algorithm 4 has performance close to the original approach of Algorithm 3 (based on Fig.8 a)), but with a far lower computational complexity.

\section{CONCLUSion}

In this paper, an energy efficient precoding method for the cooperative MIMO-AF scenario (i.e. when both relay and direct links are considered) has been proposed, based on EEoptimal source and relay precoding matrices. We have formally proved the optimality of our source and relay precoders, when treated independently, by using pseudo-convexity/convexity arguments and have relied on alternating optimization (for which the convergence has been proved) for jointly optimizing them. We have provided a closed-form expression for the EE-optimal source precoder and have designed an optimal numerical approach for obtaining the relay precoder. We have also derive a sub-optimal relay precoder in closed-form that exhibits EE performance close to the EE-optimal relay precoder, but with a far lower computational complexity. Simulation results have confirmed that our novel EE-based approach can improve the EE of cooperative MIMO-AF systems by up to $50 \%$ in comparison with existing approaches. In the future, we plan to extend our work for the case where only imperfect or partial CSI is available at the source and/or relay node, given the promising results of Fig. 7 . 


\section{APPENDIX}

\section{A. Proof of Proposition 1}

Proof: Based on the definition of [32], any function $f$ must verify the following relationship in order to be a strictly pseudo-convex function of $\mathbf{X} \succeq \mathbf{0}$,

$$
\left\langle\nabla_{\mathbf{X}} f(\mathbf{X}), \mathbf{E}\right\rangle_{\mathrm{F}} \geq 0 \Rightarrow f(\mathbf{X}+\mathbf{E})>f(\mathbf{X})
$$

for any Hermitian matrix $\mathbf{E}$ such that $\mathbf{X}+\mathbf{E} \succeq \mathbf{0}$ and $\mathbf{E} \neq \mathbf{0}$. Given that $f(\mathbf{X})=\frac{p(\mathbf{X})}{q(\mathbf{X})}$, it implies that $\left\langle\nabla_{\mathbf{X}} f(\mathbf{X}), \mathbf{E}\right\rangle_{\mathrm{F}}=$

$$
q(\mathbf{X})^{-1}\left[\left\langle\nabla_{\mathbf{X}} p(\mathbf{X}), \mathbf{E}\right\rangle_{\mathrm{F}}-f(\mathbf{X})\left\langle\nabla_{\mathbf{X}} q(\mathbf{X}), \mathbf{E}\right\rangle_{\mathrm{F}}\right]
$$

In turn, based on (41), $\left\langle\nabla_{\mathbf{X}} f(\mathbf{X}), \mathbf{E}\right\rangle_{\mathrm{F}} \geq 0$ is equivalent to

$$
\left\langle\nabla_{\mathbf{X}} p(\mathbf{X}), \mathbf{E}\right\rangle_{\mathrm{F}} \geq f(\mathbf{X})\left\langle\nabla_{\mathbf{X}} q(\mathbf{X}), \mathbf{E}\right\rangle_{\mathrm{F}}
$$

Based on the definition of [33], $q$ is a strictly concave function of $\mathbf{X} \succeq \mathbf{0}$ if and only if

$$
\left\langle\nabla_{\mathbf{X}} q(\mathbf{X}), \mathbf{E}\right\rangle_{\mathrm{F}}>q(\mathbf{X}+\mathbf{E})-q(\mathbf{X})
$$

for any Hermitian matrix $\mathbf{E}$ such that $\mathbf{X}+\mathbf{E} \succeq \mathbf{0}$ and $\mathbf{E} \neq \mathbf{0}$. By inserting equation (43) into (42), we obtain

$$
\begin{aligned}
& \left\langle\nabla_{\mathbf{X}} p(\mathbf{X}), \mathbf{E}\right\rangle_{\mathrm{F}}>f(\mathbf{X})[q(\mathbf{X}+\mathbf{E})-q(\mathbf{X})] \\
\Leftrightarrow & q(\mathbf{X})\left\langle\nabla_{\mathbf{X}} p(\mathbf{X}), \mathbf{E}\right\rangle_{\mathrm{F}}>p(\mathbf{X})[q(\mathbf{X}+\mathbf{E})-q(\mathbf{X})] \\
\Leftrightarrow & q(\mathbf{X})\left[p(\mathbf{X})+\left\langle\nabla_{\mathbf{X}} p(\mathbf{X}), \mathbf{E}\right\rangle_{\mathrm{F}}\right]>p(\mathbf{X}) q(\mathbf{X}+\mathbf{E}) .
\end{aligned}
$$

Given that $p$ is a linear function of $\mathbf{X}$, it implies that $p(\mathbf{X}+$ $\mathbf{E})=p(\mathbf{X})+\left\langle\nabla_{\mathbf{X}} p(\mathbf{X}), \mathbf{E}\right\rangle_{\mathrm{F}}$. Consequently,

$$
\begin{aligned}
& q(\mathbf{X}) p(\mathbf{X}+\mathbf{E})>p(\mathbf{X}) q(\mathbf{X}+\mathbf{E}) \\
\Leftrightarrow & \frac{p(\mathbf{X}+\mathbf{E})}{q(\mathbf{X}+\mathbf{E})}>\frac{p(\mathbf{X})}{q(\mathbf{X})} \Leftrightarrow f(\mathbf{X}+\mathbf{E})>f(\mathbf{X}) .
\end{aligned}
$$

Hence, (40) is verified and $f(\mathbf{X})$ is strictly pseudo-convex.

\section{B. Proof of Proposition 2}

Proof: Let $q$ be a strictly pseudo-convex function, such that it verifies (40), and $p$ be a function of $\mathbf{X} \succeq \mathbf{0}$; hence,

$$
\begin{aligned}
& (p(\mathbf{X}+\mathbf{E})-p(\mathbf{X}))+\left\langle\nabla_{\mathbf{X}} q(\mathbf{X}), \mathbf{E}\right\rangle_{\mathrm{F}} \geq 0 \Rightarrow \\
& (p(\mathbf{X}+\mathbf{E})-p(\mathbf{X}))+q(\mathbf{X}+\mathbf{E})-q(\mathbf{X})>0 \\
\Leftrightarrow & (p(\mathbf{X}+\mathbf{E})-p(\mathbf{X}))+\left\langle\nabla_{\mathbf{X}} q(\mathbf{X}), \mathbf{E}\right\rangle_{\mathrm{F}} \geq 0 \Rightarrow \\
& p(\mathbf{X}+\mathbf{E})+q(\mathbf{X}+\mathbf{E})>p(\mathbf{X})+q(\mathbf{X}) .
\end{aligned}
$$

Let $f(\mathbf{X})=p(\mathbf{X})+q(\mathbf{X})$ and $p$ be linear, i.e. $p(\mathbf{X}+\mathbf{E})=$ $p(\mathbf{X})+\left\langle\nabla_{\mathbf{X}} p(\mathbf{X}), \mathbf{E}\right\rangle_{\mathrm{F}}$, it then implies that

$$
\begin{aligned}
& \left\langle\nabla_{\mathbf{X}} p(\mathbf{X}), \mathbf{E}\right\rangle_{\mathrm{F}}+\left\langle\nabla_{\mathbf{X}} q(\mathbf{X}), \mathbf{E}\right\rangle_{\mathrm{F}} \geq 0 \Rightarrow f(\mathbf{X}+\mathbf{E})>f(\mathbf{X}) \\
\Leftrightarrow & \left\langle\nabla_{\mathbf{X}} f(\mathbf{X}), \mathbf{E}\right\rangle_{\mathrm{F}} \geq 0 \Rightarrow f(\mathbf{X}+\mathbf{E})>f(\mathbf{X}),
\end{aligned}
$$

which is the definition of a strictly pseudo-convex function in (40).
C. Proof of Corollary 1: Strict Positivity and Concavity of (16a)

Proof: On the one hand, let $R_{\Sigma}(\mathbf{Y})$ be defined as in (16a), it then implies that $R_{\Sigma}(\mathbf{Y})>0$ for $\mathbf{Y} \succeq \mathbf{0}, \mathbf{Y} \neq \mathbf{0}$. Moreover, by relying on matrix calculus [37], it also implies that

$$
\left\langle\nabla_{\mathbf{Y}} R_{\Sigma}(\mathbf{Y}), \mathbf{E}\right\rangle_{\mathrm{F}}=W / \ln (2) \operatorname{tr}\left\{\left(\mathbf{I}_{n_{\mathrm{SN}}}+\mathbf{Y}\right)^{-1} \mathbf{E}\right\} .
$$

Note that $\left(\mathbf{I}_{n_{\mathrm{SN}}}+\mathbf{Y}\right)^{\dagger}=\left(\mathbf{I}_{n_{\mathrm{SN}}}+\mathbf{Y}\right)$ since $\left(\mathbf{I}_{n_{\mathrm{SN}}}+\mathbf{Y}\right)$ is a Hermitian matrix. On the other hand,

$$
R_{\Sigma}(\mathbf{Y}+\mathbf{E})-R_{\Sigma}(\mathbf{Y})=W \log _{2}\left|\mathbf{I}_{n_{\mathrm{SN}}}+\left(\mathbf{I}_{n_{\mathrm{SN}}}+\mathbf{Y}\right)^{-1} \mathbf{E}\right|
$$

Knowing that for any square matrix $\mathbf{X},\left|e^{\mathbf{X}}\right|=e^{\operatorname{tr}\{\mathbf{X}\}} \Leftrightarrow$ $\operatorname{tr}\{\mathbf{X}\}=\ln \left|e^{\mathbf{X}}\right|$, and by using the change of variable $\mathbf{X}_{\mathbf{1}}=\left(\mathbf{I}_{n_{\mathrm{SN}}}+\mathbf{Y}\right)^{-1} \mathbf{E} \succ \mathbf{0}$ (when assuming that $\mathbf{E} \neq$ $\mathbf{0})$, (48) and (49) can be re-expressed as $W \log _{2}\left|e^{\mathbf{X}_{1}}\right|$ and $W \log _{2}\left|\mathbf{I}_{n_{\mathrm{SN}}}+\mathbf{X}_{\mathbf{1}}\right|$, respectively. Since $\left|e^{\mathbf{X}_{\mathbf{1}}}\right|>\left|\mathbf{I}_{n_{\mathrm{SN}}}+\mathbf{X}_{\mathbf{1}}\right|$ for any square matrix $\mathbf{X}_{\mathbf{1}} \succ \mathbf{0}, R_{\Sigma}$ verifies (43) and, in turn, is a strictly positive and concave function of $\mathbf{Y}$.

\section{Proof of Proposition 4}

Proof: The Lagrangian associated with the optimization problem in (15) is formulated in (17). Given that, according to Corollary 1 , this function is strictly pseudo-convex or convex, it implies that the global optimum of $\mathcal{L}\left(\mathbf{Y}, \lambda_{1}, \lambda_{2}\right)$ occurs at a stationary point, such that

$$
\nabla_{\mathbf{Y}} \mathcal{L}\left(\mathbf{Y}=\mathbf{Y}^{\star}, \lambda_{1}^{\star}, \lambda_{2}^{\star}\right)=\mathbf{0}
$$

where $\lambda_{i}^{\star}=\lambda_{i} R_{\Sigma}\left(\mathbf{Y}^{\star}\right), \forall i \in\{1,2\}$.

1) Unconstrained Optimization: According to the first equation of (17), (50) is equivalent to

$$
\nabla_{\mathbf{Y}} P_{\Sigma}\left(\mathbf{Y}=\mathbf{Y}^{\star}\right)-E_{b}^{\star} \nabla_{\mathbf{Y}} R_{\Sigma}\left(\mathbf{Y}=\mathbf{Y}^{\star}\right)=\mathbf{0},
$$

in the unconstrained case, where $E_{b}^{\star}=E_{b}\left(\mathbf{Y}^{\star}\right)=\frac{P_{\Sigma}\left(\mathbf{Y}^{\star}\right)}{R_{\Sigma}\left(\mathbf{Y}^{\star}\right)}$. According to the definitions of $R_{\Sigma}(\mathbf{Y})$ in (16a) and $P_{\Sigma}(\mathbf{Y})=$ $P_{c}^{\prime}+\operatorname{tr}\left\{\mathbf{Y} \Psi\left(\Delta_{\mathrm{SN}}, \Delta_{\mathrm{RN}}\right)\right\}$ in the first line of (17), it then implies, by relying on matrix calculus [37], that

$$
\begin{aligned}
& \nabla_{\mathbf{Y}} P_{\Sigma}(\mathbf{Y})=\mathbf{\Psi}\left(\Delta_{\mathrm{SN}}, \Delta_{\mathrm{RN}}\right) \text { and } \\
& \nabla_{\mathbf{Y}} R_{\Sigma}(\mathbf{Y})=\frac{W}{\ln (2)}\left(\mathbf{I}_{n_{\mathrm{SN}}}+\mathbf{Y}\right)^{-1},
\end{aligned}
$$

respectively. By inserting the results in (52) into (51), the latter can be re-expressed as

$$
\begin{aligned}
& \frac{W E_{b}^{\star}}{\ln (2)} \mathbf{I}_{n_{\mathrm{SN}}}=\boldsymbol{\Psi}\left(\Delta_{\mathrm{SN}}, \Delta_{\mathrm{RN}}\right)\left[\mathbf{I}_{n_{\mathrm{SN}}}+\mathbf{Y}^{\star}\right] \\
\Leftrightarrow & \mathbf{Y}^{\star}=\frac{W E_{b}^{\star}}{\ln (2)} \boldsymbol{\Psi}(\alpha, \beta)^{-1}-\mathbf{I}_{n_{\mathrm{SN}}},
\end{aligned}
$$

where $\alpha=\Delta_{\mathrm{SN}}$ and $\beta=\Delta_{\mathrm{RN}}$.

2) Single Power Constrained Optimization: According to the second and third equations of (17), (50) is equivalent to

$$
\begin{aligned}
& \boldsymbol{\Psi}\left(0, \Delta_{\mathrm{RN}}\right)-\frac{W E_{b}^{\star}}{\ln (2)}\left(\mathbf{I}_{n_{\mathrm{SN}}}+\mathbf{Y}^{\star}\right)^{-1}+\mathbf{\Psi}\left(\lambda_{1}^{\star}, 0\right)=\mathbf{0} \text { and } \\
& \boldsymbol{\Psi}\left(\Delta_{\mathrm{SN}}, 0\right)-\frac{W E_{b}^{\star}}{\ln (2)}\left(\mathbf{I}_{n_{\mathrm{SN}}}+\mathbf{Y}^{\star}\right)^{-1}+\boldsymbol{\Psi}\left(0, \lambda_{2}^{\star}\right)=\mathbf{0},
\end{aligned}
$$


in the SPC case at the SN and SPC case at the RN, respectively. Equivalently,

$$
\begin{aligned}
& \frac{W E_{b}^{\star}}{\ln (2)} \mathbf{I}_{n_{\mathrm{SN}}}=\mathbf{\Psi}\left(\lambda_{1}^{\star}, \Delta_{\mathrm{RN}}\right)\left[\mathbf{I}_{n_{\mathrm{SN}}}+\mathbf{Y}^{\star}\right] \text { and } \\
& \frac{W E_{b}^{\star}}{\ln (2)} \mathbf{I}_{n_{\mathrm{SN}}}=\mathbf{\Psi}\left(\Delta_{\mathrm{SN}}, \lambda_{2}^{\star}\right)\left[\mathbf{I}_{n_{\mathrm{SN}}}+\mathbf{Y}^{\star}\right]
\end{aligned}
$$

such that equality (53b) holds, but where $\alpha=\lambda_{1}^{\star}$ and $\beta=\Delta_{\mathrm{RN}}$ in the SPC case at the $\mathrm{SN}$, and $\alpha=\Delta_{\mathrm{SN}}$ and $\beta=\lambda_{2}^{\star}$ in the SPC case at the RN.

3) Dual Power Constrained Optimization: According to the fourth equation of (17), (50) is equivalent to

$$
\begin{aligned}
& \mathbf{0}-\frac{W E_{b}^{\star}}{\ln (2)}\left(\mathbf{I}_{n_{\mathrm{SN}}}+\mathbf{Y}^{\star}\right)^{-1}+\mathbf{\Psi}\left(\lambda_{1}^{\star}, \lambda_{2}^{\star}\right)=\mathbf{0} \\
\Leftrightarrow & \frac{W E_{b}^{\star}}{\ln (2)} \mathbf{I}_{n_{\mathrm{SN}}}=\mathbf{\Psi}\left(\lambda_{1}^{\star}, \lambda_{2}^{\star}\right)\left[\mathbf{I}_{n_{\mathrm{SN}}}+\mathbf{Y}^{\star}\right],
\end{aligned}
$$

in the DPC case, such that equality (53b) holds, but where $\alpha=\lambda_{1}^{\star}$ and $\beta=\lambda_{2}^{\star}$.

Finally, since $\boldsymbol{\Psi}(\alpha, \beta)$ is a Hermitian matrix, it can decomposed as $\boldsymbol{\Psi}(\alpha, \beta)=\mathbf{U}_{\Psi} \widehat{\boldsymbol{\Psi}}(\alpha, \beta) \mathbf{U}_{\Psi}^{\dagger}$, where $\mathbf{U}_{\Psi}$ is a unitary matrix containing the eigenvectors of $\boldsymbol{\Psi}(\alpha, \beta)$ and $\widehat{\boldsymbol{\Psi}}(\alpha, \beta)=\operatorname{diag}\left(\left[\psi_{1}(\alpha, \beta), \psi_{2}(\alpha, \beta), \ldots, \psi_{n_{\mathrm{SN}}}(\alpha, \beta)\right]\right)$ is a diagonal matrix containing the eigenvalues of $\boldsymbol{\Psi}(\alpha, \beta)$. Hence, equation (53b) can be reformulated as

$$
\mathbf{Y}^{\star}=\frac{W E_{b}^{\star}}{\ln (2)}\left(\mathbf{U}_{\Psi} \widehat{\Psi}(\alpha, \beta) \mathbf{U}_{\Psi}^{\dagger}\right)^{-1}-\mathbf{I}_{n_{\mathrm{SN}}}=\mathbf{U}_{\Psi} \widehat{\mathbf{Y}}^{\star} \mathbf{U}_{\Psi}^{\dagger},
$$

where $\widehat{\mathbf{Y}}^{\star}=\frac{W E_{b}^{\star}}{\ln (2)} \widehat{\mathbf{\Psi}}(\alpha, \beta)^{-1}-\mathbf{I}_{n_{\mathrm{SN}}}$ is a diagonal matrix with elements, $y_{i}^{\star}$, as defined in (19). Note that the operator [.] $]_{+}$is used in (19) since both $\mathbf{Y} \succeq \mathbf{0}$ and $\mathbf{R} \succeq \mathbf{0}$.

\section{E. Proof of the Strict Positivity and Concavity of (26a)}

Proof: On the one hand, let $R_{\Sigma}(\mathbf{Z})$ be defined as in (26a), it then implies that $R_{\Sigma}(\mathbf{Z})>0$ since $R_{0}>0$ when $\mathbf{R} \succeq \mathbf{0}$, $\mathbf{R} \neq \mathbf{0}$. Moreover, by relying on matrix calculus [37], it also implies that $\left\langle\nabla_{\mathbf{Z}} R_{\Sigma}(\mathbf{Z}), \mathbf{E}\right\rangle_{\mathrm{F}}=$

$$
W / \ln (2) \operatorname{tr}\left\{\left[\left(\mathbf{\Upsilon}^{-1}+\mathbf{Z}\right)^{-1}-\left(\mathbf{I}_{n_{\mathrm{RN}}}+\mathbf{Z}\right)^{-1}\right] \mathbf{E}\right\} .
$$

Note that $\left(\mathbf{\Upsilon}^{-1}+\mathbf{Z}\right)^{-1} \succeq\left(\mathbf{I}_{n_{\mathrm{RN}}}+\mathbf{Z}\right)^{-1}$ given that $\boldsymbol{\Upsilon} \succeq \mathbf{I}$. On the other hand,

$$
R_{\Sigma}(\mathbf{Z}+\mathbf{E})-R_{\Sigma}(\mathbf{Z})=W \log _{2}\left|\frac{\mathbf{I}_{n_{\mathrm{RN}}}+\left(\mathbf{\Upsilon}^{-1}+\mathbf{Z}\right)^{-1} \mathbf{E}}{\mathbf{I}_{n_{\mathrm{RN}}}+\left(\mathbf{I}_{n_{\mathrm{RN}}}+\mathbf{Z}\right)^{-1} \mathbf{E}}\right| .
$$

Knowing that for any square matrix $\mathbf{X}, \operatorname{tr}\{\mathbf{X}\}=$ $\ln \left|e^{\mathbf{X}}\right|$, and by using the change of variables $\mathbf{X}_{1}=$ $\left[\left(\mathbf{\Upsilon}^{-1}+\mathbf{Z}\right)^{-1}-\left(\mathbf{I}_{n_{\mathrm{RN}}}+\mathbf{Z}\right)^{-1}\right] \mathbf{E} \succeq \mathbf{0}$ and $\mathbf{X}_{\mathbf{2}}=$ $\left(\mathbf{I}_{n_{\mathrm{RN}}}+\mathbf{Z}\right)^{-1} \mathbf{E} \succ \mathbf{0}$ (when assuming that $\mathbf{E} \neq$ 0), (58) and (59) can be re-expressed as $W \log _{2}\left|e^{\mathbf{X}_{1}}\right|$ and $W \log _{2}\left|\frac{\mathbf{I}_{n_{\mathrm{RN}}}+\mathbf{X}_{1}+\mathbf{X}_{2}}{\mathbf{I}_{n_{\mathrm{RN}}}+\mathbf{X}_{2}}\right|$, respectively. Since $\left|e^{\mathbf{X}_{1}}\right| \geq$ $\left|\mathbf{I}_{n_{\mathrm{RN}}}+\mathbf{X}_{1}\right|$ for any square matrix $\mathbf{X}_{\mathbf{1}} \succeq \mathbf{0}$ and $\left|\frac{\mathbf{I}_{n_{\mathrm{RN}}}+\mathbf{X}_{1}+\mathbf{X}_{2}}{\mathbf{I}_{n_{\mathrm{RN}}}+\mathbf{X}_{2}}\right|=\left|\mathbf{I}_{n_{\mathrm{RN}}}+\frac{\mathbf{X}_{1}}{\mathbf{I}_{n_{\mathrm{RN}}}+\mathbf{X}_{2}}\right|<\left|\mathbf{I}_{n_{\mathrm{RN}}}+\mathbf{X}_{1}\right|, R_{\Sigma}$ in (26a) verifies (43) and, in turn, is a strictly positive and concave function of $\mathbf{Z}$.

\section{F. Proof for Proposition 5}

Proof: By inserting G in (27) into (23), the denominator and numerator of the latter can be re-expressed as $R_{\Sigma}\left(\widehat{\mathbf{G}}, \mathbf{V}_{\mathbf{G}}, \mathbf{U}_{\mathbf{G}}\right)=$

$R_{0}+W \log _{2}\left|\mathbf{I}_{n_{\mathrm{RN}}}+\frac{\sigma_{1}^{2}}{\sigma_{2}^{2}} \mathbf{\Upsilon}^{\frac{1}{2}} \mathbf{U}_{\mathbf{G}} \widehat{\mathbf{G}}^{\frac{1}{2}} \mathbf{V}_{\mathbf{G}}^{\dagger} \mathbf{H}_{2}^{\dagger} \mathbf{H}_{2} \mathbf{V}_{\mathbf{G}} \widehat{\mathbf{G}}^{\frac{1}{2}} \mathbf{U}_{\mathbf{G}}^{\dagger} \mathbf{\Upsilon}^{\frac{1}{2}}\right|$

$-W \log _{2}\left|\mathbf{I}_{n_{\mathrm{RN}}}+\frac{\sigma_{1}^{2}}{\sigma_{2}^{2}} \widehat{\mathbf{G}}^{\frac{1}{2}} \mathbf{V}_{\mathbf{G}}^{\dagger} \mathbf{H}_{2}^{\dagger} \mathbf{H}_{2} \mathbf{V}_{\mathbf{G}} \widehat{\mathbf{G}}^{\frac{1}{2}}\right|$ and

$P_{\Sigma}\left(\widehat{\mathbf{G}}, \mathbf{U}_{\mathbf{G}}\right)=P_{c}^{\prime}+\frac{\sigma_{1}^{2}}{\sigma_{2}^{2}} \operatorname{tr}\left\{\widehat{\mathbf{G}} \mathbf{U}_{\mathbf{G}}^{\dagger} \ddot{\mathbf{\Upsilon}} \mathbf{U}_{\mathbf{G}}\right\}$.

Consequently, the following inequalities hold

$$
\begin{aligned}
& R_{\Sigma}\left(\widehat{\mathbf{G}}, \mathbf{V}_{\mathbf{G}}, \mathbf{U}_{\mathbf{G}}\right) \leq \max _{\mathbf{V}_{\mathbf{G}}, \mathbf{U}_{\mathbf{G}}} R_{\Sigma}\left(\widehat{\mathbf{G}}, \mathbf{V}_{\mathbf{G}}, \mathbf{U}_{\mathbf{G}}\right) \text { and } \\
& \min _{\mathbf{U}_{\mathbf{G}}} P_{\Sigma}\left(\widehat{\mathbf{G}}, \mathbf{U}_{\mathbf{G}}\right) \leq P_{\Sigma}\left(\widehat{\mathbf{G}}, \mathbf{U}_{\mathbf{G}}\right) .
\end{aligned}
$$

On the one hand, based on the work of [14] (which relies on Hadamard determinant inequality), we know that the function of the form as described in (60a) are maximized when the arguments of both determinant operators become diagonal matrices (when assuming that the eigenvalues of all the matrices are sorted in the same order). This can easily be achieved by setting $\mathbf{V}_{G_{\boldsymbol{r}}}=\mathbf{V}_{2}$ and $\mathbf{U}_{\mathbf{G}}=\mathbf{U}_{\Upsilon}$ in (60a). Hence, $\max _{\mathbf{V}_{\mathbf{G}}, \mathbf{U}_{\mathbf{G}}} R_{\Sigma}\left(\widehat{\mathbf{G}}, \mathbf{V}_{\mathbf{G}}, \mathbf{U}_{\mathbf{G}}\right)=R_{\Sigma}\left(\widehat{\mathbf{G}}, \mathbf{V}_{2}, \mathbf{U}_{\Upsilon}\right)=$

$$
R_{0}+W \log _{2}\left|\mathbf{I}_{n_{\mathrm{RN}}}+\frac{\sigma_{1}^{2}}{\sigma_{2}^{2}} \widehat{\mathbf{G}} \widehat{\boldsymbol{\Lambda}} \widehat{\boldsymbol{\Upsilon}}\right|-W \log _{2}\left|\mathbf{I}_{n_{\mathrm{RN}}}+\frac{\sigma_{1}^{2}}{\sigma_{2}^{2}} \widehat{\mathbf{G}} \widehat{\boldsymbol{\Lambda}}\right|,
$$

which is equivalent to equation (29) when $\widehat{\mathbf{Z}}=\frac{\sigma_{1}^{2}}{\sigma_{2}^{2}} \widehat{\mathbf{G}} \widehat{\mathbf{\Lambda}}$. On the other hand, the matrix $\mathbf{U}_{\mathbf{G}}$ that minimizes $P_{\Sigma}\left(\widehat{\mathbf{G}}, \mathbf{U}_{\mathbf{G}}\right)$, or equivalently $\operatorname{tr}\left\{\widehat{\mathbf{G}} \mathbf{U}_{\mathbf{G}}^{\dagger} \ddot{\mathbf{Y}} \mathbf{U}_{\mathbf{G}}\right\}$ according to (60b), is simply the matrix matching the largest values of $\widehat{\mathrm{G}}$ with the smallest values of $\mathbf{U}_{\mathbf{G}}^{\dagger} \ddot{\Upsilon} U_{\mathrm{G}}$. This can easily be achieved by setting $\mathbf{U}_{\mathbf{G}}=\mathbf{U}_{\ddot{\Upsilon}}^{\uparrow}$, where $\mathbf{U}_{\ddot{\Upsilon}}^{\uparrow}$ is unitary matrix that contains a column permutation of the eigenvectors of $\mathbf{U}_{\ddot{\Upsilon}}$, i.e. $\left(\mathbf{U}_{\ddot{\Upsilon}}^{\uparrow}\right)^{\dagger} \ddot{\Upsilon} \mathbf{U}_{\ddot{\Upsilon}}^{\uparrow}=\widehat{\ddot{\Upsilon}}^{\uparrow}$. Hence, $\widehat{\ddot{\Upsilon}}^{\uparrow}$ is a diagonal matrix containing the eigenvalues of $\ddot{\Upsilon}$ sorted in ascending order. Therefore, $\min _{\mathbf{U}_{\mathbf{G}}} P_{\Sigma}\left(\widehat{\mathbf{G}}, \mathbf{U}_{\mathbf{G}}\right)=P_{\Sigma}\left(\widehat{\mathbf{G}}, \mathbf{U}_{\ddot{\mathbf{\Upsilon}}}^{\uparrow}\right)=$

$$
P_{c}^{\prime}+\frac{\sigma_{1}^{2}}{\sigma_{2}^{2}} \operatorname{tr}\left\{\widehat{\mathbf{G}} \widehat{\ddot{\Upsilon}}^{\uparrow}\right\},
$$

which is equivalent to $P_{c}^{\prime}+\Delta_{\mathrm{RN}} P_{\mathrm{RN}}(\widehat{\mathbf{Z}})$, with $P_{\mathrm{RN}}(\widehat{\mathbf{Z}})$ as in (28) when $\widehat{\mathbf{Z}}=\frac{\sigma_{1}^{2}}{\sigma_{2}^{2}} \widehat{\mathbf{G}} \widehat{\boldsymbol{\Lambda}}$.

\section{G. Proof of Proposition 7}

Proof: The Lagrangian associated with the lower and upper bounds of the original problem in (23) can be expressed as in (30). Given that, according to Corollary 2, this function is strictly pseudo-convex or convex, it implies that the global optimum of $\mathcal{L}(\widehat{\mathbf{Z}}, \mu)$ occurs at a stationary point, such that

$$
\nabla_{\widehat{\mathbf{Z}}} \mathcal{L}\left(\widehat{\mathbf{Z}}=\widehat{\mathbf{Z}}^{\bullet}, \mu^{\bullet}\right)=\mathbf{0},
$$

where $\mu^{\bullet}=\frac{P_{c}^{\prime}+\Delta_{\mathrm{RN}} P_{\mathrm{RN}}^{\max }}{\mu\left(R_{\Sigma}\left(\widehat{\mathbf{Z}}_{\bullet}\right)\right)^{2}}$. 
1) Unconstrained Optimization: According to the first equation of (30), (64) is equivalent to

$$
\nabla_{\widehat{\mathbf{Z}}} P_{\mathrm{RN}}\left(\widehat{\mathbf{Z}}=\widehat{\mathbf{Z}}^{\bullet}\right)-E_{b}^{\bullet} \nabla_{\widehat{\mathbf{Z}}} R_{\Sigma}(\widehat{\mathbf{Z}}=\widehat{\mathbf{Z}} \bullet)=\mathbf{0},
$$

in the unconstrained case, where $E_{b}^{\bullet}=\frac{P_{\Sigma}\left(\hat{\mathbf{Z}}^{\bullet}\right)}{R_{\Sigma}\left(\widehat{\mathbf{Z}}^{\bullet}\right)}$. Based on the definitions of $R_{\Sigma}(\widehat{\mathbf{Z}})$ in (29) and $P_{\mathrm{RN}}(\widehat{\mathbf{Z}})$ in (28) or (32), it then implies, by relying on matrix calculus [37], that

$$
\begin{aligned}
& \nabla_{\widehat{\mathbf{Z}}} P_{\mathrm{RN}}(\widehat{\mathbf{Z}})=\operatorname{diag}\left\{\mathbf{u}^{\bullet}\right\} \text { and } \\
& \nabla_{\widehat{\mathbf{Z}}} R_{\Sigma}(\widehat{\mathbf{Z}})=\frac{W}{\ln (2)}\left[\left(\mathbf{\Upsilon}^{-1}+\widehat{\mathbf{Z}}\right)^{-1}-\left(\mathbf{I}_{n_{\mathrm{RN}}}+\widehat{\mathbf{Z}}\right)^{-1}\right],
\end{aligned}
$$

respectively, where $\mathbf{u}^{\bullet}$ is a vector containing either the elements of $\widehat{\Lambda}^{-1} \widehat{\hat{\Upsilon}}^{\uparrow}$ (for $P_{\mathrm{RN}}(\widehat{\mathbf{Z}})$ in (28)) or the diagonal elements of $\widehat{\boldsymbol{\Lambda}}^{-1} \ddot{\ddot{\mathbf{\Upsilon}}}$ (for $P_{\mathrm{RN}}(\widehat{\mathbf{Z}})$ in (32)). Given that $\nabla_{\widehat{\mathbf{Z}}} P_{\mathrm{RN}}(\widehat{\mathbf{Z}})$ and $\nabla_{\widehat{\mathbf{Z}}} R_{\Sigma}(\widehat{\mathbf{Z}})$ are both diagonal matrices, by inserting the results in (66) into (65), the latter can be re-expressed as

$$
\begin{gathered}
u_{i}^{\bullet}=\frac{W E_{b}^{\bullet}}{\ln (2)}\left[\frac{1}{\frac{1}{v_{i}}+z_{i}^{\bullet}}-\frac{1}{1+z_{i}^{\bullet}}\right] \\
\Leftrightarrow\left(z_{i}^{\bullet}\right)^{2} v_{i}+z_{i}^{\bullet}\left(1+v_{i}\right)+1-\frac{W \gamma\left(v_{i}-1\right)}{\ln (2) u_{i}^{\bullet}}=0,
\end{gathered}
$$

$\forall i \in\left\{1, \ldots, n_{\mathrm{RN}}\right\}$, where $\gamma=E_{b}^{\bullet}$.

2) Power Constrained Optimization: According to the second equation of (30), (64) is equivalent to

$$
\nabla_{\widehat{\mathbf{Z}}} P_{\mathrm{RN}}\left(\widehat{\mathbf{Z}}=\widehat{\mathbf{Z}}^{\bullet}\right)-\mu^{\bullet} \nabla_{\widehat{\mathbf{Z}}} R_{\Sigma}\left(\widehat{\mathbf{Z}}=\widehat{\mathbf{Z}}^{\bullet}\right)=\mathbf{0}
$$

in the power constrained case. In turn, equality (67b) holds, but where $\gamma=\mu^{\bullet}$.

Equation (33) can then be obtained by solving the quadratic equation in (67b). Note that the operator $[.]_{+}$is used in (33) to reflect the fact that both $\widehat{\mathbf{Z}} \succeq \mathbf{0}$ and $\mathbf{G} \succeq \mathbf{0}$.

\section{H. Proof for Proposition 8}

Proof: Knowing that $E_{b}$ in (23), which is a continuous function, is lower and upper bounded by continuous functions having a global minimum, it implies that $E_{b}$ has also a global minimum, $E_{b}^{\star}$ (note that $E_{b}$ can also have local extrema or saddle points), such that

$$
E_{b}^{-} \leq E_{b}^{\star} \leq E_{b}^{+} .
$$

Furthermore, we know from (p.194 of [38]) that any extrema of a differentiable function can only occur at stationary points. Hence, the global minimum of $E_{b}$ must occur at a stationary point such that $\nabla_{\mathbf{G}} E_{b}\left(\mathbf{G}=\mathbf{G}^{\star}\right)=\mathbf{0}$. According to the expression of $E_{b}(\mathbf{G})$ in (23) and matrix calculus [37], $\nabla_{\mathbf{G}} E_{b}(\mathbf{G})$ is equivalent to (37), where

$$
\begin{aligned}
\nabla_{\mathbf{G}} P_{\Sigma}(\mathbf{G}) & =\frac{2 \sigma_{1}^{2}}{\sigma_{2}^{2}} \mathbf{G} \ddot{\Upsilon} \text { and } \nabla_{\mathbf{G}} R_{\Sigma}(\mathbf{G})=\frac{2 \sigma_{1}^{2} W}{\sigma_{2}^{2} \ln (2)} \mathbf{H}_{2}^{\dagger} \\
& \times\left[\boldsymbol{\Omega}\left(\mathbf{G} \boldsymbol{\Upsilon}^{1 / 2}\right)^{-1} \mathbf{H}_{2} \mathbf{G} \boldsymbol{\Upsilon}-\boldsymbol{\Omega}(\mathbf{G})^{-1} \mathbf{H}_{2} \mathbf{G}\right] .
\end{aligned}
$$

Given that $E_{b}^{-} \leq E_{b}^{\star}$, as it is mentioned in (69), it implies that there exists a unique real nonnegative number $\varepsilon^{\star}$ such that $E_{b}^{\star}=E_{b}^{-}+\varepsilon^{\star}$ and $E_{b}^{-}+\varepsilon^{\star}$ verifies equation (36). In addition, it exists a matrix $\mathbf{G}^{\star}=\mathbf{G}\left(E_{b}^{-}+\varepsilon^{\star}\right)$ that verifies equation (37) when $E_{b}^{\star}$ is known. In other words, if $E_{b}^{\star}$ is the global minimum of $E_{b}$ occurring at $\mathbf{G}^{\star}$, then $\mathbf{G}\left(E_{b}^{-}+\varepsilon\right)$ with $\varepsilon<\varepsilon^{\star}$ can verify (37) when $E_{b}^{\star}$ is known, but $E_{b}\left(\mathbf{G}\left(E_{b}^{-}+\varepsilon\right)\right)>E_{b}^{\star}$. Thus, if $\varepsilon$ is a nonnegative real number that increases in an infinitesimal manner from 0 to infinity, the first value of $\varepsilon$ for which (36) is verified can only be the global minimum of $E_{b}$.

\section{ACKNOWLEDGMENT}

The first author wishes to thank his beloved wife for her support.

\section{REFERENCES}

[1] International Telecommunication Union (ITU), "IMT Vision Framework and overall objectives of the future development of IMT for 2020 and beyond," ITU, Geneva, Switzerland, ITU-R Recommendations M.20830, Sep. 2015.

[2] H. Zhang et al., "Energy efficiency in communications," IEEE Commun. Mag., vol. 48, no. 11, pp. 48-79, Nov. 2010.

[3] L. M. Correia et al., "Challenges and enabling technologies for energy aware mobile radio networks," IEEE Commun. Mag., vol. 48, no. 11, pp. 66-72, Nov. 2010.

[4] G. Auer et al., "How much energy is needed to run a wireless network ?" IEEE Wireless Commun., vol. 18, no. 5, pp. 40-49, Oct. 2011.

[5] A. Sendonaris, E. Erkip, and B. Aazhang, "User cooperation diversity part I \& II- system description / implementation aspects and performance analysis," IEEE Trans. Commun., vol. 51, no. 11, pp. 1927-1948, Nov. 2003.

[6] A. Nosratinia, T. E. Hunter, and A. Hedayat, "Cooperative Communication in wireless networks," IEEE Commun. Mag., vol. 42, no. 10, pp. 74-80, Oct. 2004.

[7] J. N. Laneman, D. N. C. Tse, and G. W. Wornell, "Cooperative diversity in wireless networks: efficient protocols and outage behavior," IEEE Trans. Inf. Theory, vol. 50, no. 12, pp. 3062-3080, Dec. 2004.

[8] J. Vidal et al. (2008-2009) Reconfigurable OFDMA-based Cooperative NetworKs Enabled by Agile SpecTrum Use (ROCKET). Universitat Politecnica de Catalunya and others. Barcelona, Spain. [Online]. Available: http://cordis.europa.eu/project/rcn/85296_en.html

[9] C.-L. Chen, W. E. Stark, and S.-G. Chen, "Energy-bandwidth efficiency tradeoff in MIMO multi-hop wireless networks," IEEE J. Sel. Areas Commun., vol. 29, no. 8, pp. 1537-1546, Sep. 2011.

[10] A. Zappone, P. Cao, and E. A. Jorswieck, "Energy efficiency optimization in relay-assisted MIMO systems with perfect and statistical CSI," IEEE Trans. Signal Process., vol. 62, no. 2, pp. 443-457, Jan. 2014.

[11] F. Héliot, "Low-complexity energy-efficient joint resource allocation for two-hop MIMO-AF systems," IEEE Trans. Wireless Commun., vol. 13, no. 6, pp. 3088-3099, Jun. 2014.

[12] F. Héliot and R. Tafazolli, "Optimal energy-efficient joint resource allocation for multi-hop MIMO-AF systems," IEEE Trans. Commun., vol. 64, no. 9, pp. 3655-3668, Sep. 2016.

[13] RAN WG1 (Radio layer 1), "Evolved Universal Terrestrial Radio Access (E-UTRA); Physical layer for relaying operation (Release 10-14)," The 3rd Generation Partnership Project (3GPP), Technical Specifications 36.216, 2010 onwards. [Online]. Available: http: //www.3gpp.org/dynareport/36216.htm

[14] O. Muñoz-Medina, J. Vidal, and A. Agustín, "Linear transceiver design in nonregenerative relays with channel state information," IEEE Trans. Signal Process., vol. 55, no. 6, pp. 2593-2604, Jun. 2007.

[15] I. Hammerström and A. Wittneben, "Power allocation schemes for amplify-and-forward MIMO-OFDM relay links," IEEE Trans. Wireless Commun., vol. 6, no. 8, pp. 2798-2802, Aug. 2007.

[16] R. Mo and Y. H. Chew, "Precoder design for non-regenerative MIMO relay systems," IEEE Trans. Wireless Commun., vol. 8, no. 10, pp. 50415049, Oct. 2009.

[17] F. Héliot, R. Hoshyar, and R. Tafazolli, "On the spectral efficiency maximization of nonregenerative cooperative MIMO communication systems," in Proc. IEEE PIMRC, Istanbul, Turkey, Sep. 2010.

[18] Y. Zhang, J. Li, L. Pang, and Z. Ding, "On precoder design for amplify-and-forward MIMO relay systems," in Proc. IEEE VTC-Fall, San Fransisco, CA, Sep. 2011. 
[19] N. Wang and M. Chen, "Joint source and relay precoder design for MIMO relay systems with direct link," in Proc. IEEE WCSP, Huangshan, China, Oct. 2012.

[20] Y. Rong, "Optimal joint source and relay beamforming for MIMO relays with direct link," IEEE Commun. Lett., vol. 14, no. 5, pp. 390-392, May 2010.

[21] C. Xing et al., "A general robust linear transceiver design for multihop amplify-and-forward MIMO relaying systems," IEEE Trans. Signal Process., vol. 61, no. 5, pp. 1196-1209, Mar. 2013.

[22] L. Sanguinetti, A. A. D'Amico, and Y. Rong, "A tutorial on the optimization of amplify-and-forward MIMO relay systems," IEEE $J$. Sel. Areas Commun., vol. 30, no. 8, pp. 1331-1346, Sep. 2012.

[23] F. Héliot and R. Tafazolli, "Optimal energy-efficient source and relay precoding for cooperative MIMO-AF systems," in in Proc. EuCNC, Oulu, Finland, Jun. 2017.

[24] R. Hunger et al., "Alternating optimization for MMSE broadcast precoding," in Proc. IEEE ICASSP, Toulouse, France, May 2006, pp. 757-760.

[25] Y. Rong and F. Gao, "Optimal beamforming for non-regenerative MIMO relays with direct link," IEEE Commun. Lett., vol. 13, no. 12, pp. 926928, Dec. 2009

[26] Y. Qi, F. Héliot, and M. A. Imran, "Green relay techniques in cellular systems," in Green communications and networking, F. R. Yu, X. Zhang, and V. C. Leung, Eds. Boca Raton, FL: CRC press, Dec. 2012, ch. 3.

[27] G. Miao, N. Himayat, and G. Y. Li, "Energy-efficient link adaptation in frequency-selective channels," IEEE Trans. Commun., vol. 58, no. 2, pp. 545-554, Feb. 2010.

[28] F. Héliot, M. A. Imran, and R. Tafazolli, "On the energy efficiencyspectral efficiency trade-off over the MIMO Rayleigh fading channel," IEEE Trans. Commun., vol. 60, no. 5, pp. 1345-1356, May 2012.

[29] S. Verdu, "Spectral efficiency in the wideband regime," IEEE Trans. Inf. Theory, vol. 48, no. 6, pp. 1319-1343, Jun. 2002.

[30] F. Héliot, E. Katranaras, O. Onireti, and M. A. Imran, "On the energy efficiency-spectral efficiency trade-off in cellular systems," in Green communications theoretical fundamentals algorithms and applications, J. Wu, S. Rangan, and H. Zhang, Eds. Boca Raton, FL: CRC press, Nov. 2012, ch. 17.

[31] F. Héliot, M. A. Imran, and R. Tafazolli, "Low-complexity energyefficient coordinated resource allocation in cellular systems," IEEE Trans. Commun., vol. 61, no. 6, pp. 2271-2281, Jun. 2013.

[32] M. Avriel, Nonlinear Programming: Analysis and Methods. New-York, Dover Publications, Sep. 2003.

[33] S. Boyd and L. Vandenberghe, Convex Optimization. Cambridge, UK: Cambridge Univ. Press, Mar. 2004.

[34] E. W. Weisstein, "Newton's method." MathWorld-A Wolfram Web Resource. [Online]. Available: http://mathworld.wolfram.com/ NewtonsMethod.html

[35] R. S. Prabhu and B. Daneshrad, "Energy-efficient power loading for a MIMO-SVD system and its performance in flat fading," in Proc. IEEE Globecom, Miami, FL, Dec. 2010.

[36] T. Yoo and A. Goldsmith, "Capacity and power allocation for fading MIMO channels with channel estimation error," IEEE Trans. Inf. Theory, vol. 52, no. 5, pp. 2203-2214, May 2006.

[37] K. B. Petersen and M. S. Pedersen, "The matrix cookbook," nov 2012. [Online]. Available: http://www2.imm.dtu.dk/pubdb/p.php?3274

[38] M. Golden, Mathematical Methods for Neural Network Analysis and Design. Cambridge, MA: MIT Press, Dec. 1996.

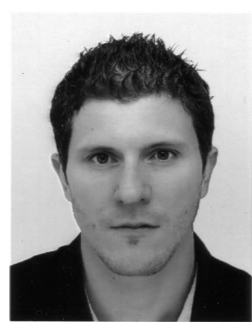

Fabien Héliot (S'05-M'07) received the MSc degree in Telecommunications from the Institut Supérieur de l'Electronique et du Numérique (ISEN), Toulon, France, and the $\mathrm{PhD}$ degree in Mobile Telecommunications from King's College London, in 2002 and 2006 , respectively. He is currently a Lecturer at the Institute for Communication Systems (ICS) of the University of Surrey, formerly known as CCSR. He has been actively involved in European Commission (EC) funded projects such as FIREWORKS, ROCKET, SMART-Net, LEXNET projects and in the award-winning EARTH project. He is currently involved in the 5GIC project, a UK funded project on shaping the future of wireless communication. His research interests include energy efficiency, EM exposure reduction, cooperative communication, MIMO, and radio resource management. He received an Exemplary Reviewer Award from IEEE COMMUNICATIONS LETTERS in 2011.

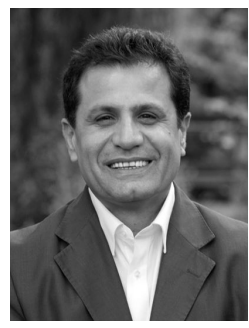

Rahim Tafazolli (SM'09) is a professor and the Director of the Institute for Communication Systems (ICS) and 5G Innovation Centre (5GIC), University of Surrey, United Kingdom. He has been active in research for more than 25 years and has authored and co-authored more than 800 papers in refereed international journals and conferences. Professor Tafazolli is a consultant to many mobile companies, has lectured, chaired, and been invited as keynote speaker to a number of IET and IEEE workshops and conferences. He has been Technical Advisor to many mobile companies, all in the field of mobile communications. He is the Founder and past Chairman of IET International Conference on 3rd Generation Mobile Communications. He is a Fellow of the IET and WWRF (Wireless World Research Forum). He is Chairman of EU Expert Group on Net!Works Technology Platform. 